\title{
Electrically tunable GHz oscillations in doped GaAs-AlAs superlattices
}

\author{
J. Kastrup⿹, R. Hey, and K.H. Ploog \\ Paul-Drude-Institut für Festkörperelektronik, \\ Hausvogteiplatz 5-7, D-10117 Berlin, Germany \\ H. T. Grahn \\ Research Center for Quantum Effect Electronics, Tokyo Institute of Technology, \\ 2-12-1 O-okayama, Meguro-ku, Tokyo 152, Japan \\ L. L. Bonilla, M. Kindelan, M. Moscoso, A. Wackere \\ Escuela Politécnica Superior, Universidad Carlos III de Madrid, \\ Butarque 15, E-28911 Leganés, Spain \\ J. Galán \\ Departamento de Matemática Aplicada II, Escuela Superior de Ingeniería, \\ Avda. Reina Mercedes s/n, 41012 Sevilla, Spain
}

\begin{abstract}
Tunable oscillatory modes of electric-field domains in doped semiconductor superlattices are reported. The experimental investigations demonstrate the realization of tunable, $\mathrm{GHz}$ frequencies in GaAs-AlAs superlattices covering the temperature region from 5 to $300 \mathrm{~K}$. The orgin of the tunable oscillatory modes is determined using an analytical and a numerical modeling of the dynamics of domain formation. Three different oscillatory modes are found. Their presence depends on the actual shape of the drift velocity curve, the doping density, the boundary condition, and the length of the superlattice. For most bias regions, the self-sustained oscillations are due to the formation, motion, and recycling of the domain boundary inside the superlattice. For some biases, the strengths of the low and high field domain change periodically in time with the domain boundary being pinned within a few quantum wells. The dependency of the frequency on the coupling leads to the prediction of a new type of tunable $\mathrm{GHz}$ oscillator based on semiconductor superlattices.
\end{abstract}

PACS numbers: 73.40.Gk, 73.50.Fq, 73.61.Ey

Typeset using REVTEX 


\section{INTRODUCTION}

Since the observation and explanation of the Gunn-effect in the early 60's it has been known that traveling field inhomogeneities are generated by negative differential velocity (NDV). theoretically and experimentally e $^{-}$leading to several proposals for applications. The most important application is probably the frequency generator. Although oscillator devices based on the Gunn-effect operating in the range of $100 \mathrm{GHz}$ have been reported, , Gunn-oscillators have not led to the expected breakthrough in technology. This is largely due to the difficulties in tuning the oscillation frequency. Furthermore, it is impossible to vary the drift velocity vs field characteristics $(v(F)$ curve) except by using different bulk materials (e.g., GaAs, InGaAs or InP).

These problems may be overcome by using an entirely different class of NDV exhibiting materials formed by semiconductor superlattices. In an applied electric field perpendicular to the two-dimensional layers, several different transport mechanisms such as miniband transport, resonant tunneling, and real-space transfer from well to barriers $(\Gamma \rightarrow X$ transport) give rise to complex $v(F)$ curves with several regions of NDV as shown in Fig. 1. In contrast to the above mentioned materials, the tunability of the barrier and well thickness as well as the control over the barrier height in the superlattice can be used to tailor the drift velocity vs field $v(F)$ characteristics. By proper engineering, samples with several NDV regions can be manufactured with control over previously inaccessible features such as the actual shape of the NDV (cf. Fig. 1). The frequency of the oscillations also depends on the number of moving charges in the superlattice, which may be controlled by varying the doping in the quantum wells.

Currently, three different types of superlattice oscillations due to NDV have to be distinguished :

- The most prominent type of oscillations is found, when carriers within the miniband are accelerated beyond the Brillouin zone boundary, where their drift velocity becomes negative. In the absence of scattering, the electron wave packet then oscillates with the Bloch frequency $f_{B}=e F d / h$, where $d$ and $h$ denote the superlattice period and Planck's constant, respectively.6-1 These so-called Bloch oscillations were predicted for superlattices by Esaki and Tsu in 1970. Inspired by the prospect of THz frequency generators based on superlattice Bloch oscillators, a long series of investigations targeting the miniband regime followed leading to the observation of damped Bloch oscillations in experiments with pulsed optical excitation a few years ago. 9

- A different type of oscillations occurs in the miniband regime, when the scattering times are shorter than the tunneling times. In this case transient charge accumulations traveling through the superlattice may lead to oscillations in the current. Experiments using pulsed optical excitation of carriers in superlattices with wide minibands showed damped oscillations with frequencies up to $20 \mathrm{GHz} 10$ More recently, reflection gain up to $60 \mathrm{GHz}$ in doped superlattices with miniband widths of up to $80 \mathrm{meV}$ was demonstrated.11

- Very recently a third type of oscillations has been shown to exist in weakly coupled superlattices in field regions where tunneling into higher subbands takes place.12 14 As 
a consequence of domain formation, this type of oscillations may also be observed in field regions where the electrons tunnel between the lowest subbands. While the first two oscillation types have never been observed under constant bias conditions, this new type is shown to operate over a broad frequency range practically independent of the external circuit and without any external triggering.

In this paper we investigate experimentally and theoretically the third type of oscillations in lightly and moderately doped superlattices (SL). This type of oscillations may be generally observable in the NDV regime of the $v(F)$ characteristics of such superlattices. In a companion paper 15 we have shown that self-sustained oscillations may exist if the dimensionless doping parameter, $\nu=e N_{D} d /\left(\epsilon F_{M}\right)\left(N_{D}\right.$ is the 3D SL doping, and $F_{M}$ is the field at the resonance we consider) lies between two values $\left(\nu_{1} / N, \nu_{2}\right)$ which can be calculated numerically from the local $v(F)$ characteristics ( $N$ is the number of SL periods). If several tunneling resonances exist (cf. Fig. 1(a) and (b)) the frequency of the oscillations increases with increasing index of the subband involved in the tunneling resonance. This observation is attributed to the larger drift velocities associated with the resonances involving higher subbands. Furthermore, several new oscillatory modes without the typically well-defined domains are shown to exist. These oscillatory modes can be tuned in frequency simply by changing the applied voltage. Because the oscillations are observable even at room temperature, we suggest to use superlattice oscillators based on resonant tunneling into higher subbands as a tunable source for high frequencies.

\section{EXPERIMENTAL RESULTS}

Four GaAs/AlAs superlattice structures, grown by molecular beam epitaxy, are discussed in this paper. The samples are hereafter referred to as 9.0/4.0 (doped and undoped), 9.0/1.5, and 13.3/2.7, where the first number refers to the GaAs well width and the second to the AlAs barrier thickness in nm (cf. Table $\mathbb{D}$ ). The superlattices are embedded between two highly doped $\mathrm{Al}_{0.5} \mathrm{Ga}_{0.5}$ As contact layers with a doping density $\mathrm{N}_{D}=2 \times 10^{18} \mathrm{~cm}^{-3}$ (Si for $n^{+}$- and Be for $p^{+}$-type doping) forming $n^{+}-n-n^{+}$and $p^{+}-i-n^{+}$diodes. After evaporating $\mathrm{AuGe} / \mathrm{Ni}$ contacts onto the top and substrate side and alloying them for ohmic connections, the samples are wet-etched into mesas of $120 \mu \mathrm{m}$ diameter. The circular top contacts of $70 \mu \mathrm{m}$ diameter leave a large part of the mesas uncovered to allow for optical access. All experiments are performed at $5 \mathrm{~K}$ in a He-flow cryostat using high-frequency coaxial cables with a bandwidth of $20 \mathrm{GHz}$. The time-averaged current-voltage data are recorded with a Keithley SMU236. The time-resolved current is detected with a Tektronix sampling oscilloscope CSA 803 using the GHz sampling head SD-32.

\section{A. Current-voltage characteristics}

Fig. 2 shows the current-voltage (I-V) characteristics of the doped 9.0/4.0 sample in forward bias. Clearly visible are two plateau regions with almost constant current between 0.5 and $4 \mathrm{~V}$ and 6.5 and $8.5 \mathrm{~V}$. To prove that these plateaus are related to the subband resonances, time-of-flight (TOF) measurements were performed on an undoped 9.0/4.0 reference sample $\left(p^{+}-i-n^{+}\right.$diode) 16 The peak photocurrent is thereby taken as a measure for 
the inverse transport time, which at low excitation densities is directly proportional to the drift velocity for homogeneous fields. Note that for the presentation in Fig. 2 the built-in voltage $(1.5 \mathrm{~V})$ of the $p^{+}-i-n^{+}$diode has been subtracted from the reverse bias voltage applied to the $p^{+}-i-n^{+}$diode and the sign has been inverted. The resonances for tunneling from the first into the second $\left(C_{1} \rightarrow C_{2}\right)$ as well as into the third $\left(C_{1} \rightarrow C_{3}\right)$ conduction subband are clearly observed at 6 and $15 \mathrm{~V}$, respectively. The low-field transmission maximum $\left(C_{1} \rightarrow C_{1}\right)$, which in the case of a strongly coupled superlattice corresponds to miniband conduction, is not resolved in this sample, because the transport time is too long in this field range to be resolved with this method. However, it has been shown previously that even weakly coupled superlattices exhibit a negative differential drift velocity in this regime. 10 In addition to the $C_{1} \rightarrow C_{j}$ resonances due to $\Gamma$-subbands in the GaAs wells and the exponential nonresonant background, a pronounced step-like increase of the peak photocurrent is observed at about $10 \mathrm{~V}$, which is assigned to enhanced transport through the lowest $X$-level in the AlAs barriers. The lowest $X$-states of the AlAs barriers are energetically located in such a way that transport through these $X$ states becomes possible at voltages between the $C_{1} \rightarrow C_{2}$ and $C_{1} \rightarrow C_{3}$ resonances. In the drift velocity vs field characteristics, this $\Gamma \rightarrow X$ resonance leads to a step-like increase of the drift velocity as indicated by the dotted line in Fig. 1(b).

The voltages of the observed resonances can be compared to the calculated energy levels listed in Table 凹. For example, the bias voltages $U_{\text {bias }}$ for $\Gamma \rightarrow \Gamma$ resonances are calculated using

$$
U_{r e s}\left(C_{1} \rightarrow C_{j}\right)=\frac{N\left(C_{j}-C_{1}\right)}{e},
$$

where $C_{1}$ denotes the energy of the injecting conduction subband and $C_{j}$ the energy of the conduction subband, in which the carriers are tunneling into. $N$ refers to the number of periods and $e$ to the electron charge. In the case of $\Gamma \rightarrow X$ resonances, however, the distance between the levels is reduced to half the superlattice period. Therefore, an additional factor of 2 has to be included, i.e.,

$$
U_{\text {res }}\left(C_{1} \rightarrow X_{j}\right)=2 \frac{N\left(X_{j}-C_{1}\right)}{e},
$$

where $X_{j}$ denotes the energy of the receiving state in the barrier. The voltages for the $\Gamma$ resonances $C_{1} \rightarrow C_{2}$ and $C_{1} \rightarrow C_{3}$ are calculated using Eq. (1D) resulting in 5.4 and $14.6 \mathrm{~V}$ for the 9.0/4.0 sample, respectively. Using Eq. (2), we obtain a resonance voltage of $8.2 \mathrm{~V}$ for the $C_{1} \rightarrow X_{1}$ resonance in this sample. All values agree well with the observed values. Note that all observed resonances appear at slightly higher voltages due to field inhomogeneities and screening effects.

To investigate the electric field inside the superlattice, photoluminescence (PL) measurements have been performed ${ }_{13}^{13} 18$ Due to the quantum-confined Stark effect (QCSE), the PL from regions with strong electric fields is red-shifted with respect to the PL from low-field regions. The experiments reveal that in both plateau regions of this sample the PL consists of two peaks with their respective intensity depending on the applied bias voltage. This Stark-splitting of the PL signal is taken as direct evidence for the existence of two distinct electric-field domains, which are formed because of NDV and current conservation. For 
the first plateau the consequence of this conservation law is depicted by the dashed line in Fig. 1(b). Clearly, the electric field of the low-field domain $\left(F_{L}\right)$ corresponds to the $C_{1} \rightarrow C_{1}$ resonance peak, while the field strength of the high field domain $\left(F_{R}\right)$ lies on the next rising branch of the $v(F)$ characteristics slightly below the $C_{1} \rightarrow C_{2}$ resonance. 1920 Applied to the second plateau, the current conservation law (dashed lines in Fig. 2) shows that the high field domain in this voltage region forms as a consequence of resonant transport through the $X_{1}$ level in the barriers $\left(C_{1} \rightarrow X_{1}\right.$ transport). In contrast to previous work, 11 where this new formation mechanism was considered for the first time, this measurements provide the first direct evidence relating $X$-levels in the barriers to domain formation. However, it should be mentioned that it is not very surprising to observe domain formation due to transport through the $X_{1}$ level. For domain formation to occur, only a minimum of the $v(F)$ curve is required in conjunction with a sufficiently high carrier density. 15 In that sense the subject of domain formation and conditions for oscillations can be discussed simply by looking at the $v(F)$ curve as discussed in a companion paper.15

In the $13.3 / 2.7$ sample the calculated $C_{1} \rightarrow X_{1}$ resonance $(13.7 \mathrm{~V})$ is located between the $C_{1} \rightarrow C_{3}$ and $C_{1} \rightarrow C_{4}$ resonances, which are calculated to be at 9.5 and $17.9 \mathrm{~V}$, respectively. Consequently, the I-V characteristic exhibits three plateaus as can be seen in Fig. 3. The thin dashed line has been added to indicate the positions of the transport maxima for homogeneous fields. Unlike in the 9.0/4.0 sample, the PL in the plateau regions shows no Stark splitting indicating that the field distribution inside the SL is less sharply defined. A possible explanation of this behavior is the existence of rather broad resonances in this weakly doped superlattice. However the I-V characteristics shows some clear signs of domain formation (e.g., current branches 1922 ), but the differences between the electric field values of these domains seem to be rather small. Since the QCSE is quadratic in the electric field, the peaks of the two domains cannot be resolved in the PL experiments. Because stable electric field domains have been investigated intensively over the last years, we will not discuss the stationary regime any further. We will rather focus on the non-stationary behavior instead.

\section{B. Oscillations in different plateaus}

The absence of a regular pattern of current branches in the plateaus is a first hint that the field distribution inside the SL may not always be stable. Indeed, the current, e.g., in the lower plateau of the 9.0/4.0 sample (Fig. Đ), shows a strongly time-dependent component, which has been attributed to an oscillatory instability of the field distribution inside the superlattice.12.13 During one cycle of the oscillation a monopole forms near the cathode, drifts through the superlattice towards the anode and dissolves, while a new monopole is formed. 13 This type of oscillation is denoted the first oscillation mode.

It is interesting to note that the displayed trace also contains a number of small current spikes. Supported by simulations, voltage turn-on measurements have shown that these small spikes are due to well-to-well hopping of the domain boundary moving through the superlattice.23 Thus, the number of current spikes indicates how many superlattice periods are involved in the oscillation. For the displayed oscillation this leads to a number close to thirty, which allows to speculate that for this particular voltage the domain boundary crosses about three quarters of the superlattice during one cycle of the oscillation. 
To analyze the oscillations in more detail, we have measured the voltage dependence of the oscillation frequency within the plateaus. The Fourier transform 12 exhibits a series of frequency maxima, of which we take the fundamental frequency as the oscillation frequency of the field distribution.

\section{Voltage independent frequency}

The voltage dependence of the oscillation frequency in the two plateaus of the 9.0/4.0 sample is plotted in Fig. 5 showing that the observed frequencies in the first plateau are weakly voltage dependent, while in the second plateau there is no dependence on the applied voltage at all. The noticeable increase of the frequency with voltage at the end of the first plateau is due to the charge monopole having less room to travel from the beginning of the superlattice to the position of the high field domain. In both plateaus there are regions, where sinusoidal oscillations could not be observed. In these regions the current contains a time dependent component, which is not periodic and therefore difficult to measure. In particular the discontinuities in the frequency at the beginning of the first plateau suggest that the oscillations may become chaotic, when the charge monopole oscillates near the contacts. While chaotic behavior has been shown to exist theoretically in the case of an external driving frequency, 24 25 the boundary regions of the plateaus are still under investigation in this respect. Very recently driven and undriven chaos have been observed in this system.26 Note that chaotic behavior has also been observed at the onset of trap-dominated Gunn oscillations in ultra-pure p-Ge.27 This behavior is due to intermittent switching between small and large amplitude current oscillations, 27 although its theoretical interpretation remains uncertain.28

\section{Voltage dependent frequency}

In contrast to the 9.0/4.0 sample, the frequencies of the oscillations in the $13.3 / 2.7$ sample exhibit a pronounced voltage dependence as shown in Fig. 6 for three different temperatures in the third plateau. The oscillations begin to appear close to the resonance maximum and decrease monotonically with increasing voltage. Furthermore, in this sample oscillations are only detected near the beginning of the plateaus in the region of NDV. This observation not only applies to the third plateau, but also to the first and second plateaus. Thus, there is a clear difference between the oscillations observed in these two samples. Unlike in the 9.0/4.0 sample, the time-resolved PL does not show an oscillating two-domain solution, 13 but merely an intensity modulated broad line. These two differences are taken as a signature for the existence of a different oscillatory mode in this sample, which is not related to well-defined electric field domains.

In Fig. 7 the experimental results on the 9.0/1.5 sample are shown. In this case we used a $25 \mu \mathrm{m}$ mesa in order to keep the current at a reasonable level. Due to the stronger coupling, $\mathrm{GHz}$ oscillations are already observed in the lowest plateau. In addition to a strong voltage dependence of the frequency, there is also an abrupt jump to lower values. Higher plateaus could not be measured due to the large increase in current at about $8 \mathrm{~V}$. Time-resolved PL spectra on a $120 \mu \mathrm{m}$ mesa do not show two well-separated lines. However, intensity 
oscillations with the same frequencies are clearly observed. Current oscillations on these large mesas are also measured using pulsed electric fields to avoid the heating of the sample.

\section{Temperature dependence}

Fig. 3 shows the current voltage characteristic of the $13.3 / 2.7$ sample at room temperature. From the fact that it still shows the plateau-like features related to resonant tunneling one can speculate that the oscillations may also be found at higher temperatures. In Fig. 6 the oscillations in the third plateau are observed up to $200 \mathrm{~K}$, while in Fig. 8 tunable oscillations indeed exist even at $295 \mathrm{~K}$ in the case of the second plateau. These results are very encouraging, because they prove that a device such as a frequency generator based on the recently found tunable oscillatory mode can in principle be manufactured. However, in order to obtain well-defined resonances even at room temperature, one probably has to switch to another material system with stronger confinement. For example, superlattices based on InGaAs/InAlAs material system exhibit pronounced plateaus even at room temperature.29 Therefore, it is expected that with properly chosen parameters oscillators based on InGaAs/InAlAs superlattices can be operated at room temperature.

\section{THEORETICAL INTERPRETATION: THREE OSCILLATORY MODES}

In order to explain why the frequency depends in one case on the applied voltage, while it is voltage independent in the other case, the condition for the two oscillatory modes has to be investigated in more detail. Numerical calculations on the following discrete drift model have been performed for the electric field $F_{i}$ and 3D electron density $\tilde{n}_{i}$ of the $i^{\text {th }}$ SL period as well as for the total current density $J$ using the following equations, 2 , 13, , 30,31

$$
\begin{array}{r}
F_{i}-F_{i-1}=\frac{e d}{\epsilon}\left(\tilde{n}_{i}-N_{D}\right), \\
\epsilon \frac{d F_{i}}{d \tilde{t}}+e \tilde{v}\left(F_{i}\right) \tilde{n}_{i}=J, \\
\frac{1}{N} \sum_{i=1}^{N} F_{i}=\frac{\Phi}{N d} .
\end{array}
$$

Here $i=1, \ldots, N$ is the period index, $N_{D}$ the 3D doping density, $d$ the SL period, $\epsilon$ the average permittivity, and $\Phi$ the applied voltage between the two SL ends. Eqn. (3)-(5) have to be supplemented using the appropriate initial conditions for the field profile $F_{i}$ as well as with a boundary condition for $F_{0}$, which we take as

$$
F_{1}(t)-F_{0}(t)=c \frac{e d N_{D}}{\epsilon}
$$

where $c>0$ denotes a dimensionless charge accumulation within the first SL period due to the excess doping outside the SL $\left(n^{+}-n-n^{+}\right.$structure). We expect that the narrower the barriers are, the larger $c>0$ is, since the overlap between wavefunctions of adjacent quantum wells is larger for narrower barriers. To find a precise relation between the structure of the $n^{+}$region before the SL and $c$, microscopic modeling of the full $n^{+}-n-n^{+}$structure 
(characterized in Ref. 20) is needed. This is outside the scope of the present work. However, we can estimate the values of $c$ by comparing experimental and calculated frequency versus voltage bias curves. Thus comparing Figs. 5, 6 and 7 with Fig. 13(b), we obtain that $c$ should be very small $(c<0.001)$ for samples $9.0 / 4.0$ and $13.3 / 2.7$, whereas $c$ should be from 10 to 100 times larger for sample 9.0/1.5 (see the discussion below).

The $\tilde{v}(F)$ function is a phenomenological electron velocity, which is proportional to the tunneling probability. The main transport mechanism in weakly coupled superlattices is sequential resonant tunneling (SRT). Thus, $\tilde{v}(F)$ has peaks at the applied voltages or electric field strengths corresponding to the alignment of the energy levels of adjacent quantum wells as given in Eq. (1), which are always separated by a region of NDV. The experimentally observed voltage dependence of the frequency in samples 9.0/4.0 and 13.3/2.7 (cf. Figs. 5 and 6) can be explained in terms of the actual shape of the $\tilde{v}(F)$ curve, the doping density in the wells and the charge accumulation within the first period. The crucial features in the $\tilde{v}(F)$ curve are the separation between the peaks as well as their width. The position of the peaks can be approximated by Eq. (1). The width of the peaks and the values of the minima of $\tilde{v}(F)$ depend on the particular scattering mechanisms, which are present in the samples. However, it is reasonable to assume that the peaks of the $\tilde{v}(F)$ curve for the $13.3 / 2.7$ sample are wider than those of the 9.0/4.0 sample, since the narrower the barriers, the wider the energy intervals for resonant tunneling. 32 Therefore, we will compare simulations of the same model using two different $\tilde{v}(F)$ curves and different doping densities. We shall use curves $\tilde{v}(F)$ with only one peak, corresponding to a $C_{1} \rightarrow C_{i}$ resonance, with $i=1,2, \ldots$ Thus we are only dealing with the self-sustained oscillations occurring in the plateau after the chosen resonance. Adding more peaks does not appreciably change the self-sustained oscillations on each different plateau, although new features may appear for highly doped SLs. In fact, there are stationary nonuniform solution branches with electric field profiles presenting simultaneous coexistence of three or more domains if a $\tilde{v}(F)$ curve with several peaks is considered. 18,33

To compare experimental results belonging to three different samples to our data, it is convenient to render the Eqs. (3)-(6) dimensionless. Suppose we want to analyze timeperiodic current oscillations at a certain plateau of the current-voltage characteristic. We adopt as the units of electric field and velocity the corresponding values at the peak of the velocity curve $\tilde{v}(F)$ prior to the plateau, $F_{M}$ and $\tilde{v}_{M}$, respectively. Thus, we have to scale the magnitudes in the figures by a different factor for each sample and plateau. The time scale factors, $s_{t}$, for the 9.0/4.0 (second plateau, after the $C_{1} \rightarrow C_{2}$ resonance), 13.3/2.7 (second plateau, after the $C_{1} \rightarrow C_{2}$ resonance), and 9.0/1.5 (first plateau, after the $C_{1} \rightarrow C_{1}$ peak), samples are 3.57, 0.98, and $0.30 \mathrm{~ns}$, respectively. The electric field scale factors, $s_{E}$, are on the same order of magnitude, $10^{5}, 4.4 \times 10^{4}$, and $2.4 \times 10^{4} \mathrm{~V} / \mathrm{cm}$. We set 15

$$
\begin{array}{lll}
E_{i}=\frac{F_{i}}{F_{M}}, & n_{i}=\frac{\tilde{n}_{i}}{N_{D}}, & I=\frac{\tilde{J}}{e N_{D} \tilde{v}_{M}}, \\
v=\frac{\tilde{v}}{\tilde{v}_{M}}, & t=\frac{\nu \tilde{v}_{M} \tilde{t}}{d}, & \phi=\frac{\Phi}{N F_{M} d},
\end{array}
$$

where the dimensionless parameter $\nu$ is defined as

$$
\nu=\frac{e N_{D} d}{\epsilon F_{M}} .
$$


A typical self-sustained current oscillation (far from the voltage bias corresponding to the onset or the end of the instability) is caused by the generation, motion and annihilation of domain walls, which are charge monopoles. 11 We want to estimate the dependence of the oscillation frequency or period $T_{p}$ on the bias $\phi$. There exist three different oscillation modes depending on the value of the bias:

- First mode: Oscillation of the electric field profile around an almost uniform stationary state (Fig. 9(a)). It occurs when the bias is just above the onset of the instability. It can be shown that the oscillation frequency decreases as the bias increases by numerically solving the problem of linear stability of the stationary electric field profile as discussed in a companion paper.15

- Second mode: Monopole recycling (Fig. 9(b)), which typically occurs when the bias is larger. In this mode of oscillation, the period is the sum of the formation time of a monopole (the time it takes each new charge accumulation injected at the first SL period to form a monopole) plus the time elapsed until another charge accumulation is injected. New monopoles are formed when the current is close to its maximum value ( $I \approx 1$, or $\tilde{J} \approx e N_{D} \tilde{v}_{M}$ in dimensional units) and the monopole formation time is small compared to the period of the oscillation. The bias interval, over which the second oscillatory mode exists, can be shown to increase with sample doping or with the number of SL periods.

- Third mode: Oscillation of the electric field profile about a nonuniform stationary state containing two electric field domains (Fig. 9(c)). This mode is sometimes present in weakly doped samples, which oscillate for larger biases. It then occurs near the end of the oscillatory branch. In this third mode of oscillation, a domain wall remains pinned at a given location and the field values at the low and high field domains oscillate in antiphase. The shape of the current oscillation is almost sinusoidal and the maximum current is clearly below 1 (where the accumulation layers were injected from the injecting contact for the mode discussed before). A similar oscillation mode has been found in simulations of imperfect superlattices 34

Next we report the results of numerical simulations of the self-sustained oscillations of the current in the discrete model for different values of the dimensionless parameters. The simulations yield self-sustained current-oscillations when the doping $\nu$ is such that the corresponding stationary field profile is clearly inhomogeneous, e.g., as in Fig. 10. The current on the middle branch of stationary solutions is significantly larger than $v(\phi)$. In these cases the bias has to be within a certain interval $\left(\phi_{\alpha}, \phi_{\omega}\right)\left(\phi_{\alpha}>1\right.$ is on the NDV branch of $v(E)$, and $\phi_{\omega}$ may or may not be larger than the minimum $\left.E_{m}\right)$ to generate self-sustained current oscillations. Both $\phi_{\alpha}>1$ and $\phi_{\omega}$ are increasing functions of $c$. In Fig. 10(a), the oscillatory behavior begins at $\phi=\phi_{\alpha} \approx 1.100$ via a supercritical Hopf bifurcation. The amplitude of the oscillation increases with bias following a square-root law and the frequency is almost constant. At $\phi=\phi_{\omega} \approx 1.617$ the branch of oscillatory solutions disappears via a second Hopf bifurcation. For longer SLs with $N=200$ and the same doping, Fig. 10(e), or for a 50-well SL with larger doping, 10)(c), the end of the oscillatory branch is different: a limit cycle collides with the unstable fixed point from the middle branch of the Z-shaped current-voltage characteristics and disappears. This bifurcation scenario 
results in a decrease of the frequency down to zero, when the collision takes place, while the amplitude is unchanged to lowest order. The bias interval where the oscillatory branch exists, $\left(\phi_{\alpha}, \phi_{\omega}\right)$, shrinks as $\nu$ decreases, and for $\nu<\nu_{0}\left(\nu_{0} \approx 0.073\right.$ for $\left.N=50, c=10^{-4}\right)$, there is no oscillatory solution. Close to the Hopf bifurcations the first and third modes of oscillation are found. We no longer see recycling and motion of domain walls. Instead, in all these examples, there is an interval of bistability between the self-sustained oscillations and the stable stationary lower branch of the Z-shaped characteristic when $\phi \in\left(\phi_{\beta}, \phi_{\omega}\right)$. The bistability may be hard to observe experimentally because the basin of attraction of the self-sustained current oscillations is very small and most initial field profiles evolve to stationary situations. A similar type of bistability can be found when (i) wiggles in the static current-voltage characteristic $I^{*}(\phi)$ appear, and (ii) the upper branch of a wiggle is unstable against oscillatory behavior while the lower branch is stable. See Fig. 1 of Ref. 15 for $\nu=0.3$. Fig. 10 shows the variation in magnitude and frequency of the current oscillations as a function of the bias for different doping values and number of SL periods.

To clarify the nature of the oscillations let us describe the second mode of oscillations, which occurs for most biases. Fig. 11(a) shows the field profiles at different times of a given period of the current oscillations for a 50-well SL. We can identify a field profile consisting of two well-formed field domains at time (1). We define the field of the domain to be the electric field $E_{i}$ at the position where $n_{i}$ has a local minimum, i.e., the variation in the field is minimal. The domain wall between two domains is a charge monopole containing an excess of electrons $\left(n_{i}>1\right)$. Its evolution towards the receiving contact (until it disappears) is captured by snapshots at times (2) to (4) in Fig. 11(a). Let $j(t)$ be the instantaneous position of the domain wall defined as the SL period for which $n_{i}$ is a local maximum. Except for the time intervals, in which two domain walls are present simultaneously, the constant voltage condition implies that

$$
E_{l}(t)=E_{h}(t)-\frac{N}{j(t)}\left(E_{h}(t)-\phi\right),
$$

where $E_{l}$ and $E_{h}$ are the values of the field in the low and high field domains, respectively. Within the low field domain there is a tiny inhomogeneity (contact layer) close to the injecting contact, which is due to the boundary condition. 5 . As long as the low field domain is in the region of positive differential velocity $\left(E_{l}<1\right)$, the field profile is stable and the contact layer follows adiabatically the current. The position $j(t)$ of the domain wall moves to the right with a certain velocity $v_{m o n} / \nu$, where $v_{m o n}$ is always significantly less than 1 (the peak velocity in dimensionless units). Eq. (9) implies that $E_{l}, E_{h}$, and $I$ must increase with time in order to fulfill the fixed voltage condition. When $E_{l}$ surpasses 1 , a small charge accumulation is injected at $i=1$, which moves to the right and grows until it becomes a wellformed charge monopole. See the snapshots at times (3) and (4) in Fig. 11(a). The process is repeated in time after the old monopole has disappeared and the new one is well established. Note that two different monopoles are present simultaneously during a certain part of the oscillation period. For a long SL the displacement current is quite small during most of each oscillation period, and we have $E_{l} \approx E^{(1)}(I)$ and $E_{h} \approx E^{(3)}(I)\left[E^{(1)}(I)<E^{(2)}(I)<E^{(3)}(I)\right.$ are the three solutions of the equation $v(E)=I$ for $I<1$. Note that the solutions $E^{(1)}$ and $E^{(2)}$ disappear for $\left.I>1\right]$. See Fig. 12 for a 200-period SL. Notice that now the domains are well-established at times for which the values of the current are different from those depicted 
in Fig. 11(a). Even for a smaller SL as the one in Fig. 11, the displacement current is small, when the current is near its maximum value for each period [see the time intervals between (1) and (2) in Fig. 11(b) and between (2) and (3) in Fig. 12(b)]. Notice that the field value on the low field domain is near the peak of the $v(E)$ curve during these time intervals. This will be useful in what follows.

\section{A. Dependence of the oscillation frequency with voltage}

It is important to understand the bias dependence of the frequency which could be used to tune the frequency of an eventual device in agreement with the experimental observations of Section II. The frequency might increase or decrease with bias depending on the sample parameters $N$ and $\nu$, as shown in Figs. 10(b,d,f). Let us start at the time $t=t_{0}$ when $E_{1}=1+c \nu$ [then $\left.v\left(E_{0}\right)=1>v\left(E_{1}\right), E_{l} \approx 1\right]$, the contact layer looses its stability, and a charge accumulation is injected at $i=1\left(t_{0} \approx 129\right.$ in Fig. 12). One period of duration $T_{p}$ is completed at $t=t_{0}+T_{p}$ when the next charge accumulation is injected. At this instant, the position of the charge monopole is given by Eq. (9) with $E_{l}=1$ and $E_{h} \approx E^{(3)}(1)$ which yields

$$
M:=j\left(t_{0}+T_{p}\right)=\frac{E^{(3)}(1)-\phi}{E^{(3)}(1)-1} N
$$

Thus, the charge accumulation has to travel a distance $M$ in the time $T_{p}$. During the monopole formation time $T_{f}, E_{l} \approx 1$, and the mean velocity of the charge accumulation is $\nu^{-1}$. On the other hand, the same charge accumulation moves at a smaller mean velocity equal to $v_{m o n} / \nu$ (with $v_{m o n}<1$ ) for the rest of the period, $T_{p}-T_{f}$, once it has become a charge monopole. We therefore have

$$
M=\frac{1}{\nu} T_{f}+\frac{v_{m o n}}{\nu}\left(T_{p}-T_{f}\right)
$$

This gives

$$
T_{p}=\frac{\nu M}{v_{m o n}}-T_{f}\left(\frac{1}{v_{m o n}}-1\right)
$$

We now estimate the monopole formation time $T_{f}$. The new charge accumulation $\delta n$ travels with a velocity of the order of $v\left(E_{l}\right) / \nu \approx 1 / \nu$ and is amplified in time via

$$
\frac{d \delta n(t)}{d t}=-v^{\prime}\left(E_{l}\right) \delta n(t)
$$

with an initial value $\delta n\left(t_{0}\right) \sim c$. At time (3) of Fig. 12, $t_{0}+T_{f}, \delta n$ is so large that it separates two different well-formed field domains. Then we may consider that a new charge monopole has been born. This charge monopole travels at the velocity $v_{m o n} / \nu$ mentioned above and it sharpens as it moves; see the point marked by (4) in the Fig. 12(a). This stage lasts until the fields before and after the monopole reach the values $E=E^{(1)}(I)$ and $E=E^{(3)}(I)$, respectively, and we are back to situation (1) having completed one 
period. Mathematically, this behavior can be well described by an asymptotic analysis in the continuum limit $(\nu \rightarrow 0, N \nu=$ const $) .35$

In order to estimate $T_{f}$, we note that it is determined by the condition $\delta n\left(t_{0}+\right.$ $\left.T_{f}\right) / \delta n\left(t_{0}\right)=a$ where $a \sim 1 / c$. Then Eq. (13) yields

$$
\log (a)=-\int_{t_{0}}^{t_{0}+T_{f}} v^{\prime}\left(E_{l}(t)\right) d t
$$

Now we obtain $E_{l}(t)$ from Eq. (9) with $E_{h} \approx E^{(3)}(1), j(t)=M+v_{\text {mon }}\left(t-t_{0}\right) / \nu$. Up to the first order in $\left(t-t_{0}\right)$, this yields

$$
E_{l}(t)=1+\frac{N v_{m o n}\left[E^{(3)}(1)-\phi\right]}{\nu M^{2}}\left(t-t_{0}\right) .
$$

Linearizing $v^{\prime}\left(E_{l}\right) \approx-\left|v^{\prime \prime}(1)\right|\left(E_{l}-1\right)$, we obtain

$$
T_{f}=\sqrt{\nu M} \cdot \sqrt{\frac{2 M \log (a)}{N v_{m o n}\left|v^{\prime \prime}(1)\right|\left[E^{(3)}(1)-1\right]}} .
$$

Now $M$ decreases as $\phi$ increases, which can be seen from Eq. (10). As $v_{\text {mon }}<1$, Eq. (12) indicates that there are two competing mechanisms influencing the dependence of $T_{p}$ with $\phi$. In general the monopole formation time $T_{f}$ is negligible compared to $T_{p}(\phi)$ in the following cases: (i) for long SLs ( $M$ in Eq. (12) is then large), (ii) when the values of $c$ are large (i.e., $a$ small), (iii) for large doping $\nu$. In these cases, $T_{p}(\phi)$ should be a decreasing function. We find that the frequency increases with $\phi$ for large values of the quantities $N, \nu$, and $c$, while it decreases otherwise. These behaviors are illustrated in Figs. 10 and 13.

We can now interpret the experimental observations according to our theoretical results.

- Sample 9.0/4.0 has narrow peaks, high doping with respect to the others, and is short $(\mathrm{N}=40)$. Therefore, the second oscillation mode dominates for most biases with a short stage of monopole formation $\left(\left|v^{\prime \prime}(1)\right|\right.$ is quite large). During most of each oscillation period, the electric field on the low field domain takes on values close to that of the resonant peak. This makes it possible to distinguish two peaks in the PL data. 2 , 30 . 36 The frequency is practically independent on the bias. 12 This sample is modeled by the velocity curve shown in the inset of Fig. 14(a). The results of the numerical simulation for current oscillations and field profiles are also shown in Fig. 14(a).

- Sample 13.3/2.7 has wide peaks, low doping, and is also short $(N=50)$. We would expect the first mode of oscillation to dominate, which agrees with the experimental observation that the frequency is a decreasing function of the bias. In this mode the high field domain is never well established, and the low field domain is off-resonance during most parts of each oscillation period (cf. Fig. 14(b)). This explains that no Stark-splitting of the PL signal is observed in the experiments for this sample.

- Sample 9.0/1.5 is similar to sample 13.3/2.7, except that its doping is 2.5 times larger and the SL is shorter $(N=40)$. The velocity curve used in the simulations for this sample is shown in the inset of Fig. 10(d). For this sample, Fig. 7 seems to indicate that the first two oscillation modes are important: for low bias the first mode dominates, 
whereas for larger bias the second mode does. Fig. I also shows that the frequency increases with bias abruptly after reaching its minimum value. Numerical calculations suggest that this could correspond to having a larger excess charge in the first SL period than in the case of sample 9.0/4.0. This could be expected for a SL with narrower barriers (cf. Fig. 13).

\section{B. Self-sustained oscillations for SL with depletion contact layers}

Until now we have studied Eqs. (3) - (5) with the boundary condition of Eq. (6) and $c$ being positive. Now we are going to describe what happens if $-1<c<0$, i.e., there are less electrons in the first well than the doping density $\left(n_{1}-1=c<0\right.$; cf. Fig. 15).

As in the case of positive $c$, the oscillations are due to the generation, motion and annihilation of domain walls connecting domains (which are regions of almost uniform electric field approximately given by the zeros of $I-v(E)$ ). The difference is that now the electric field profile is a monotone decreasing function of the QW index: the high-field domain is close to the beginning of the SL and the low-field domain extends to the end of the SL. The domain walls are now charge-depletion layers, having less electrons than the doping density. Let us describe one period of the current oscillations for a long SL as the one in Fig. 15 with $N=200$. We will assume that initially (point marked with 1 in Fig. 15(a)) the field profile has two domains connected by a domain wall $\left(E=E^{(3)}\left(I_{0}\right)\right.$ to the left of the domain wall and $E=E^{(1)}\left(I_{0}\right)$ to the right of the domain wall with an initial value of the current $I_{0} \in\left(v_{m}, 1\right)$ ). The domain wall is approximately centered at $j / N=Y=\left(\phi-E^{(1)}\left(I_{0}\right)\right) /\left(E^{(3)}\left(I_{0}\right)-E^{(1)}\left(I_{0}\right)\right)$ so that the voltage bias condition of Eq. (5) holds. The domain wall then moves towards the end of the superlattice with a speed close to the instantaneous value of the current. The current decreases until a certain minimum value slightly smaller than $v_{m}$ is reached. Then a new high-field domain is created (close to the beginning of the SL) and the current rises sharply as the two domain walls move towards the end of the SL. When the current is near its maximum value, the old domain wall disappears as the values of the field in the intermediate and rightmost domains coalesce. Then one period of the oscillation is completed. All these features can be understood by means of an asymptotic analysis to be reported elsewhere. 37 Some of the basic ideas were sketched in Ref. 31 .

\section{THE SUPERLATTICE OSCILLATOR AS A TUNABLE HIGH-FREQUENCY GENERATOR}

Clearly, a superlattice oscillator based on the above described types of oscillations may be exploited in a device, e.g., by operating the superlattice as a frequency generator. In order to establish the use of the newly found oscillations, it is important to know what maximum frequency can be achieved. While experimental investigations in this direction are still missing, it is clear that the ultimate frequency limit will be connected with the maximum drift velocity, which can be achieved without destroying the shape of the $v(F)$ curve.

To first order we assume that the oscillation frequency is determined only by the transition time of the carriers through the sample. This approach neglects a possible oscillation 
of the domain boundary over a few periods only, but allows to give an estimate for the oscillation frequencies from the current density $j$, the $3 \mathrm{D}$ doping density $N_{D}$, and the length $L=N d$ of the sample using

$$
f=\frac{j}{e L N_{D}} .
$$

In Fig. 16 the measured oscillation frequencies are plotted versus the frequencies calculated using Eq. (17). For each plateau in the 9.0/4.0 sample only one measured frequency is shown, while for the 13.3/2.7 and 9.0/1.5 samples the measured minimum and maximum frequencies are plotted. The figure shows a strong correlation between drift velocity and oscillation frequency over several orders of magnitude. The highest observed miniband drift velocities

are on the order of $10^{7} \mathrm{~cm} / \mathrm{s}$ which, as Sibille et al 38 pointed out, for a superlattice of $500 \mathrm{~nm}$ length translates into a fundamental frequency of $200 \mathrm{GHz}$. However, the electric fields in the reported case cannot be increased arbitrarily without breaking up the miniband, while for the subband field-oscillator this limitation does not apply. Accordingly, the observed frequencies become larger for higher subband resonances, which for sufficiently thin barriers will push the drift velocities and the frequencies to new limits. Looking at the oscillation frequency as a function of barrier width, we note that a change of a factor of 2.67 in barrier width leads to a change in the oscillation frequency in the first plateau of a factor of about 4000. Projecting this behavior for other material systems to feasible barrier widths, we conclude that the frequencies could reach the $100 \mathrm{GHz}$ range.

Hence, in contrast to Gunn-effect devices, the oscillation frequency of the superlattice oscillator is not only defined by the length of the sample, but also by the parameters of the heterostructure. In this respect the superlattice oscillator leaves much more room for optimization. Additionally, a frequency generator based on a superlattice oscillator with several plateaus may cover several orders of magnitude with one device. In particular the tunability within the plateaus at high frequencies allows to envision a tunable frequency generator based on resonant carrier transport covering the whole $\mathrm{GHz}$ regime, which to our knowledge no other device is capable of.

\section{SUMMARY AND CONCLUSIONS}

We have demonstrated that doped, weakly coupled semiconductor superlattices exhibit current self-oscillations with $\mathrm{GHz}$ frequencies, which can be tuned by simply changing the applied voltage. The oscillations, which are observed in GaAs-AlAs superlattices, are not limited to low temperatures, but in one case have been observed at room temperature. They occur due to an unstable electric-field domain formation. A theoretical modeling, both analytical and numerical, demonstrates that three different oscillatory modes can occur, which depend on the detailed shape of the drift velocity curve, the doping density, the boundary condition, and the length of the superlattice. In contrast to the Bloch-oscillator proposed by Esaki and Tsu, 8 the self-oscillations of the field distribution are in most cases due to a formation, motion, and recycling of a charge accumulation layer inside the superlattice. In some cases the domain boundary is localized over a few quantum wells, while the field strengths of the low and high field domain oscillate. We would like to stress that the observed oscillations are also different from the damped miniband oscillations observed by Le Person 
et al.,10 because they occur only at field strengths, where the miniband is already destroyed by Wannier-Stark localization.

Considered from a macroscopic point of view in the sense that a traveling charge accumulation layer generates the oscillation in the current, the oscillations in doped superlattices are similar to the moving field domains in Gunn diodes. In both cases the nonlinearity, which is responsible for the instability, is generated by negative differential velocity. The microscopic origin, however, is entirely different with important consequences for the frequency limit and the tunability.

In conclusion, the subband superlattice oscillator has great potential for applications as a tunable $\mathrm{GHz}$ oscillator. In order to achieve such a device, it is necessary to fully understand the origin of the oscillations and determine all possible oscillation modes. Furthermore, other material systems such as InGaAs/InAlAs should be investigated in order to generate even higher $\mathrm{GHz}$ oscillations at room temperature.

\section{ACKNOWLEDGMENTS}

The authors would like to thank D. Bertram for the time-of-flight experiments, R. Klann and S. Venakides for valuable discussions, A. Fischer for sample growth, and E. Wiebecke and H. Kostial for sample preparation. The work was supported in part by the DFG within the framework of Sfb 296, by the DGICYT grant PB94-0375, and by the EU Human Capital and Mobility Programme contract ERBCHRXCT930413. One of us (AW) acknowledges financial support from the Deutsche Forschungsgemeinschaft (DFG). 


\section{REFERENCES}

a) present address: CompuNet GmbH, Hörselbergstr. 7 D-81677 München, Germany.

b) permanent address: Paul-Drude-Institut für Festkörperelektronik, Hausvogteiplatz 5-7, D-10117 Berlin, Germany.

c) present address: Mikroelektronik Centret, Technical University of Denmark, DK-2800 Lyngby, Denmark.

${ }^{1}$ B. K. Ridley and T. B. Watkins, Proc. Phys. Soc. 78, 293 (1961).

2 J. B. Gunn, Solid State Commun. 1, 88 (1963).

${ }^{3}$ M. Shur, GaAs devices and circuits (Plenum Press, New York, 1987), p. 173.

${ }^{4}$ F. J. Higuera and L. L. Bonilla, Physica D 57, 164 (1992).

${ }^{5}$ T. G. Ruttan, Electron. Lett. 11, 294 (1975).

${ }^{6}$ F. Bloch, Z. Phys. 52, 555 (1928).

${ }^{7}$ C. Zener, Proc. R. Soc. London Ser. A 145, 523 (1934).

${ }^{8}$ L. Esaki and R. Tsu, IBM J. Res. Develop. 14, 61 (1970).

${ }^{9}$ J. Feldmann et al., Phys. Rev. B 46, 7252 (1992).

${ }^{10}$ H. Le Person, C. Minot, L. Boni, J. F. Palmier, and F. Mollot, Appl. Phys. Lett. 60, 2397 (1992).

${ }^{11}$ M. Hadjazi, J. F. Palmier, A. Sibille, H. Wang, E. Paris, and F. Mollot, Electron. Lett. 27, 648 (1993).

12 J. Kastrup et al., Phys. Rev. B 52, 13761 (1995).

${ }^{13}$ H. T. Grahn et al., Jpn. J. Appl. Phys. 34, 4526 (1995).

14 J. Kastrup, H. T. Grahn, K. Ploog, and R. Merlin, Solid-State Electron. 40, 157 (1996).

${ }^{15}$ A. Wacker, M. Moscoso, M. Kindelan, and L. L. Bonilla, Phys. Rev. B 55, 2466 (1997).

${ }^{16}$ H. Schneider, K. von Klitzing, and K. Ploog, Europhys. Lett. 8, 75 (1989).

${ }^{17}$ H. T. Grahn, K. von Klitzing, K. Ploog, and G.H. Döhler, Phys. Rev. B 43, 12094 (1991).

${ }^{18}$ H. T. Grahn, H. Schneider, and K. von Klitzing, Phys. Rev. B 41, 2890 (1990).

${ }^{19}$ H. T. Grahn, R. J. Haug, W. Müller, and K. Ploog, Phys. Rev. Lett. 67, 1618 (1991).

${ }^{20}$ S. H. Kwok, R. Merlin, H. T. Grahn, and K. Ploog, Phys. Rev. B 50, 2007 (1994).

${ }^{21}$ Y. Zhang, X. Yang, W. Liu, P. Zhang, and D. Jiang, Appl. Phys. Lett. 65, 1148 (1994).

22 J. Kastrup et al., Appl. Phys. Lett. 65, 1808 (1994).

${ }^{23}$ J. Kastrup, F. Prengel, H.T. Grahn, K. Ploog, and E. Schöll, Phys. Rev. B 53, 1502 (1996).

${ }^{24}$ O. M. Bulashenko and L. L. Bonilla, Phys. Rev. B 52, 7849 (1995).

${ }^{25}$ O. M. Bulashenko, M. J. García, and L. L. Bonilla, Phys. Rev. B 53, 10008 (1996).

${ }^{26}$ Y. Zhang, J. Kastrup, R. Klann, K. H. Ploog, and H. T. Grahn, Phys. Rev. Lett. 77, 3001 (1996).

27 A. M. Kahn, D. J. Mar, and R. M. Westervelt, Phys. Rev. B 46, 7469 (1992).

${ }^{28}$ M. J. Bergmann, S. W. Teitsworth, L. L. Bonilla, and I. R. Cantalapiedra, Phys. Rev. B 53, 1327 (1996).

${ }^{29}$ Y. Kawamura, K. Wakita, and K. Oe, Jpn. J. Appl. Phys. 26, L1603 (1987).

${ }^{30}$ L. L. Bonilla, J. Galán, J. A. Cuesta, F. C. Martínez, and J. M. Molera, Phys. Rev. B 50, 8644 (1994).

${ }^{31}$ L. L. Bonilla, in Nonlinear Dynamics and Pattern Formation in Semiconductors and Devices, edited by F.-J. Niedernostheide (Springer Verlag, Berlin, 1995), Chap. 1.

32 J. Iñarrea and G. Platero, Phys. Rev. B 51, 5244 (1995). 
${ }^{33}$ Yu. A. Mityagin, V. N. Murzin, Yu. A. Efimov and G. K. Rasulova, Superlattices Microstructures 20, in press (1996).

${ }^{34}$ A. Wacker, G. Schwarz, F. Prengel, E. Schöll, J. Kastrup, and H.T. Grahn, Phys. Rev. B 52, 13788 (1995).

${ }^{35}$ L. L. Bonilla, M. Kindelan, M. Moscoso, and S. Venakides, SIAM J. Appl. Math. 57(6), in press, cond-mat/9611239.

${ }^{36}$ S. H. Kwok et al., Phys. Rev. B 51, 10171 (1995).

${ }^{37}$ L. L. Bonilla, M. Kindelan, M. Moscoso, and A. Wacker, (unpublished).

${ }^{38}$ A. Sibille, J. F. Palmier, H. Wang, and F. Mollot, Phys. Rev. Lett. 64, 52 (1990). 


\section{FIGURES}

FIG. 1. (a) Conduction band profile of a superlattice with three subbands in an applied electric field. (b) Drift velocity vs field characteristics of this superlattice consisting of the nonresonant background, the low-field maximum $\left(C_{1} \rightarrow C_{1}\right)$, and the first two resonant tunneling maxima. The dotted line indicates a possible shape of the $v(F)$ curve in the case of a $\Gamma \rightarrow X$ resonance located between the $C_{1} \rightarrow C_{2}$ and $C_{1} \rightarrow C_{3}$ resonances. The dashed line is explained in the text.

FIG. 2. Current-voltage characteristics of the doped 9.0/4.0 sample and peak photocurrent vs applied voltage obtained by time-of-flight experiments in the undoped 9.0/4 reference sample. The dashed line indicates the condition of current conservation for the second plateau assuming a low-field domain with a field strength corresponding to the $C_{1} \rightarrow C_{2}$ resonance.

FIG. 3. Current-voltage characteristics at 4 and $295 \mathrm{~K}$ for the $13.3 / 2.7$ sample. The dashed line shows schematically the expected drift velocity vs field characteristic of this sample for a homogeneous field.

FIG. 4. Current self-oscillations at $0.78 \mathrm{~V}$ in the first plateau of the doped 9.0/4.0 sample at $4 \mathrm{~K}$.

FIG. 5. Voltage dependence of the oscillation frequency within the first (a) and second (b) plateau of the doped 9.0/4.0 sample at $4 \mathrm{~K}$. The frequencies are indicated as open circles. For comparison the time-averaged I-V characteristics are included labeled by dots.

FIG. 6. Oscillation frequency vs applied voltage in the third plateau of the $13.3 / 2.7$ sample for three different temperatures.

FIG. 7. Voltage dependence of the oscillation frequency in the 9.0/1.5 sample at $4 \mathrm{~K}$. The frequencies are indicated as open circles. For comparison the time-averaged I-V characteristic is included labeled by dots.

FIG. 8. Oscillation frequency (open circles) and amplitude (squares) vs applied voltage in the 13.3/2.7 sample at room temperature in the second plateau.

FIG. 9. Current oscillations (left) and field profiles (right) for $\nu=0.1, c=10^{-4}, N=50$ and different values of $\phi=1.10$ (a), 1.25 (b) and 1.61 (c). Note that near the extrema of the current oscillations, the field distribution is close to the one of the stationary state. 
FIG. 10. (a) Stationary current-voltage characteristic (full line) with maximum and minimum of the oscillating current (dotted lines) $N=50$. The oscillatory branch begins at $\phi_{\alpha} \approx 1.100$ and ends at $\phi_{\omega} \approx 1.617$; the interval of bistability begins at $\phi_{\beta} \approx 1.487$. (b) Fundamental frequency of the current vs. the average electric field (bias) for $N=50$. (c,d) Same as (a,b) but now $\nu=0.15$; $\phi_{\alpha} \approx 1.052, \phi_{\beta} \approx 1.556, \phi_{\omega} \approx 1.797$. (e,f) Same as (a,b) but $N=200 ; \phi_{\alpha} \approx 1.02, \phi_{\beta} \approx 1.622$, $\phi_{\omega} \approx 1.868$. In all cases $c=10^{-4}$.

FIG. 11. (a) Electric field profiles at different times during one period of the current oscillation depicted in the inset. (b) Time evolution of the electric field values in the left, middle (when it exists) and right domain of the SL. The corresponding values of $E^{(i)}(I(t)), i=1,2,3$, are represented with dashed lines. Parameter values are $\phi=1.25, c=10^{-4}, \nu=0.1$, and $N=50$.

FIG. 12. Same as in Figure 11 for $N=200$.

FIG. 13. (a) Frequency vs. bias diagrams for different values of the dimensionless doping $\nu$ and $c=0.001$. (b) Frequency vs. bias diagrams for different values of $c$ keeping $\nu=0.1$ and $N=50$ fixed.

FIG. 14. Current oscillations (left) and field profiles (right) simulating samples 9.0/4.0 (a) and $13.3 / 2.7(\mathrm{~b})$.

FIG. 15. (a) Current density versus time when $c=-0.01$. Electron density (b) and electric field (c) profiles during one period of the current oscillation. The numbers in (b) and (c) correspond to the times marked in (a). Parameter values are $\phi=1.25, \nu=0.35$, and $N=200$.

FIG. 16. Calculated vs measured frequencies for the different plateaus in the doped samples. 1 p, 2p, and 3 p denote the first, second, and third plateau, respectively. For the 13.3/2.7 and 9.0/1.5 samples the minimum and maximum frequencies for each plateau are shown. 


\section{TABLES}

TABLE I. Parameters of the four investigated samples. $N$ denotes the number of periods and $N_{2 D}$ the two-dimensional carrier density introduced by doping. $C_{i}$ and $X_{1}$ indicate the energy positions of the lowest $\Gamma$-subbands in the wells and the lowest $X$-subbands in the barriers, respectively. The width of the lowest conduction miniband is given by $\Delta_{1}$. The energies of the subbands measured from the bottom of the GaAs conduction band have been obtained by conventional envelope function calculations.

\begin{tabular}{ccccccccc}
\hline \hline $\begin{array}{c}d_{\text {GaAs }} / d_{\text {AlAs }} \\
(\mathrm{nm} / \mathrm{nm})\end{array}$ & $N$ & type & $\begin{array}{c}N_{2 D} \\
\left(\mathrm{~cm}^{-2}\right)\end{array}$ & $\begin{array}{c}C_{1} \\
(\mathrm{meV})\end{array}$ & $\begin{array}{c}C_{2} \\
(\mathrm{meV})\end{array}$ & $\begin{array}{c}C_{3} \\
(\mathrm{meV})\end{array}$ & $\begin{array}{c}X_{1} \\
(\mathrm{meV})\end{array}$ & $\begin{array}{c}\Delta_{1} \\
(\mathrm{meV})\end{array}$ \\
\hline $13.3 / 2.7$ & 50 & $n^{+}-n-n^{+}$ & $1 \times 10^{10}$ & 23 & 94 & 212 & 159 & 0.13 \\
$9.0 / 4.0$ & 40 & $n^{+}-n-n^{+}$ & $1.5 \times 10^{11}$ & 44 & 180 & 410 & 146 & $<0.1$ \\
$9.0 / 4.0$ & 40 & $p^{+}-i-n^{+}$ & - & 44 & 178 & 410 & 146 & $<0.1$ \\
$9.0 / 1.5$ & 40 & $n^{+}-n-n^{+}$ & $2.5 \times 10^{10}$ & 44 & 180 & 411 & 199 & 3.7 \\
\hline \hline
\end{tabular}


(a)
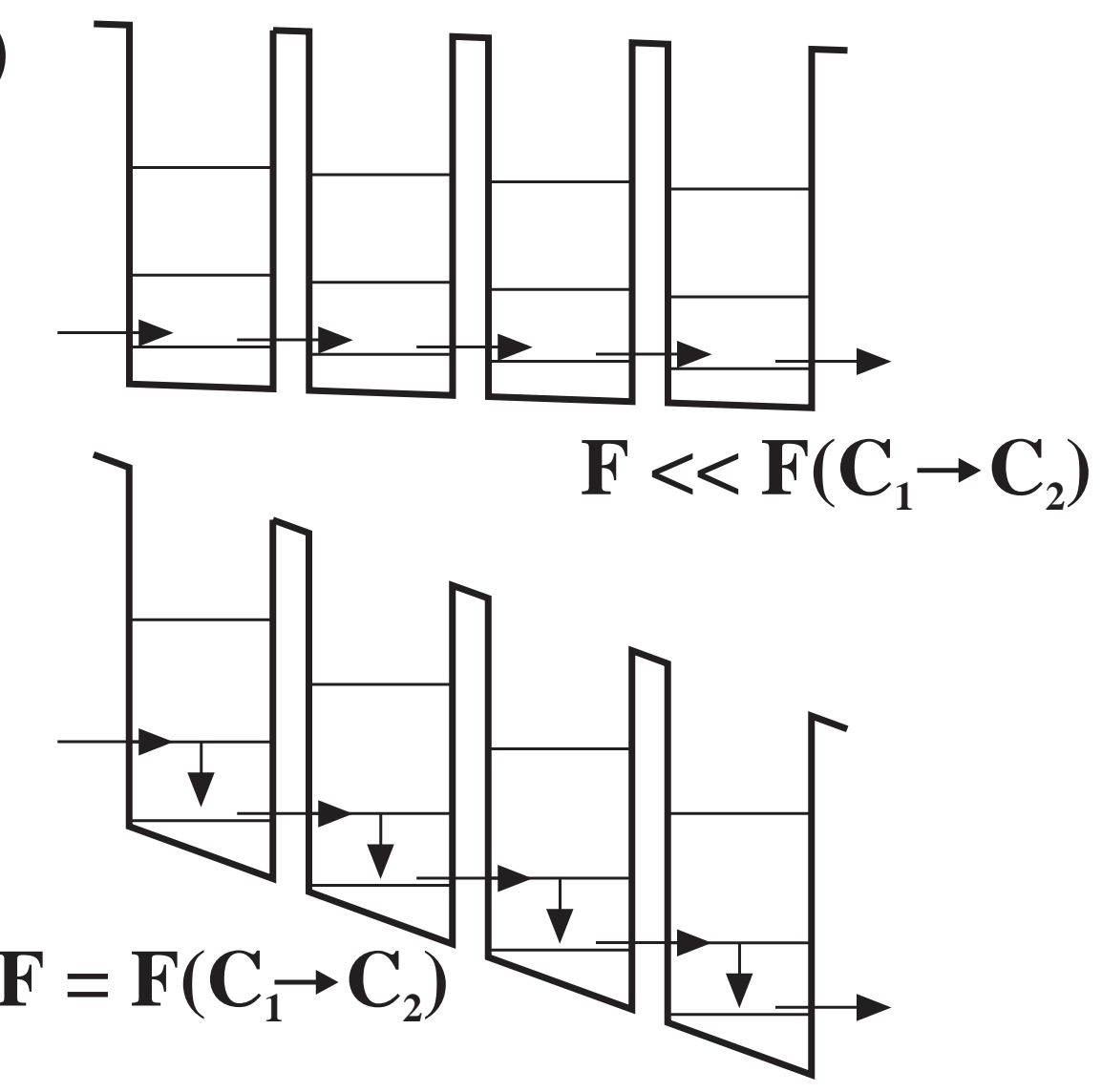

(b)

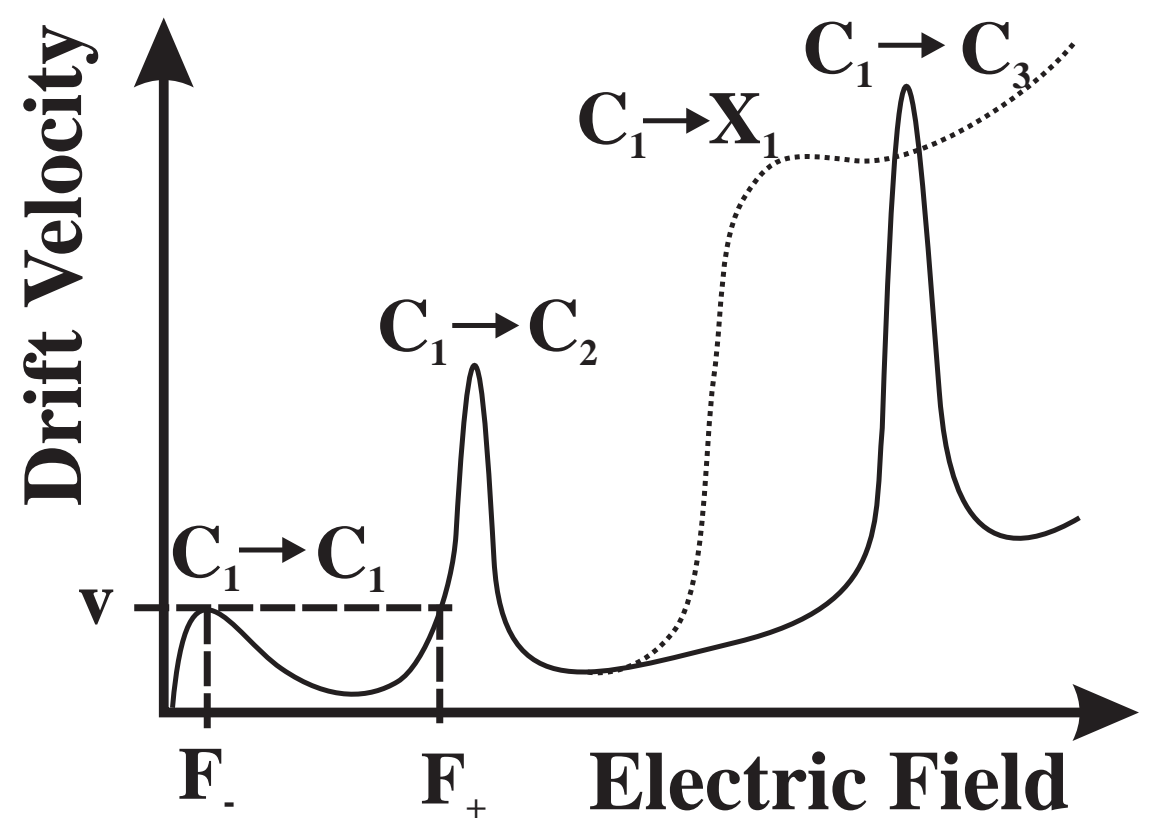

Fig. 1 


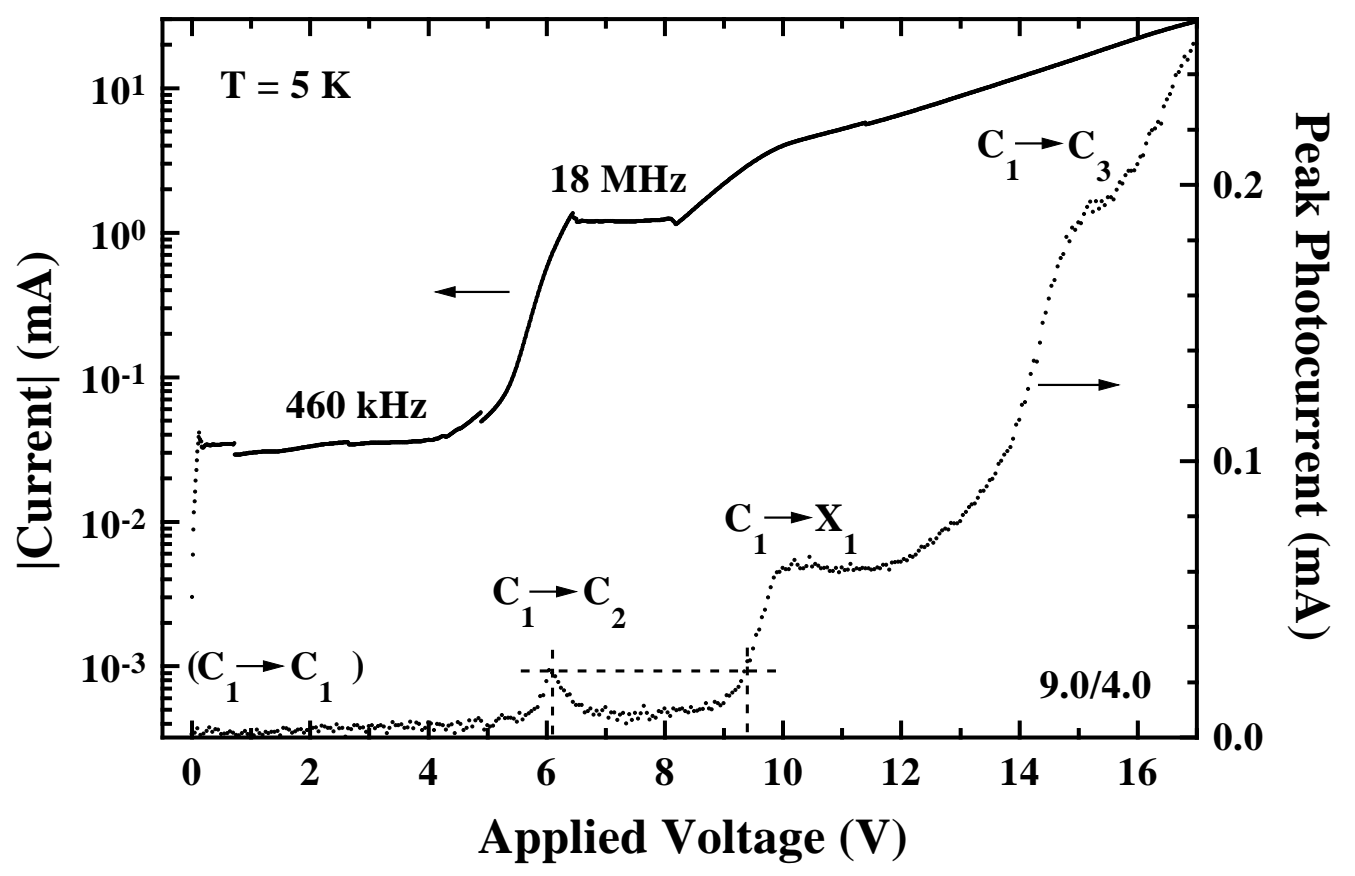

Fig. 2 


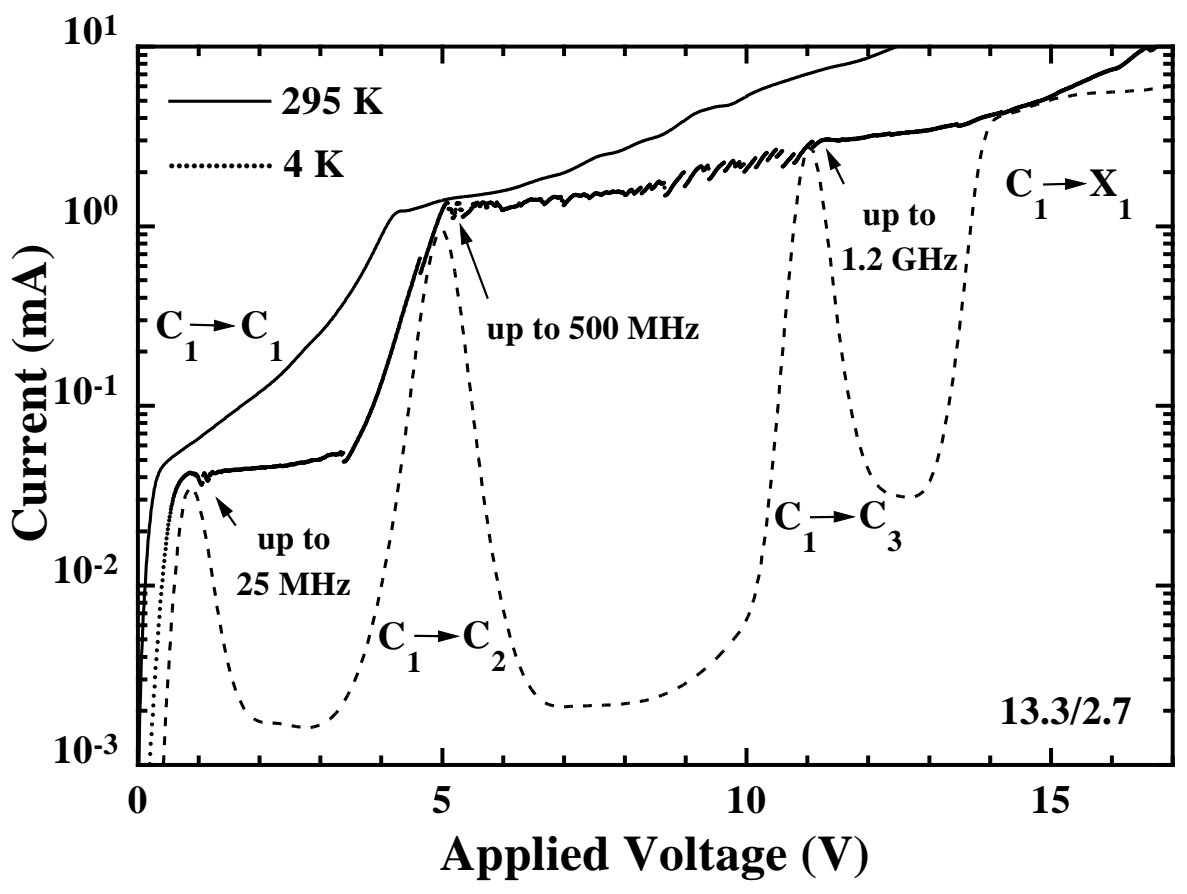

Fig. 3 


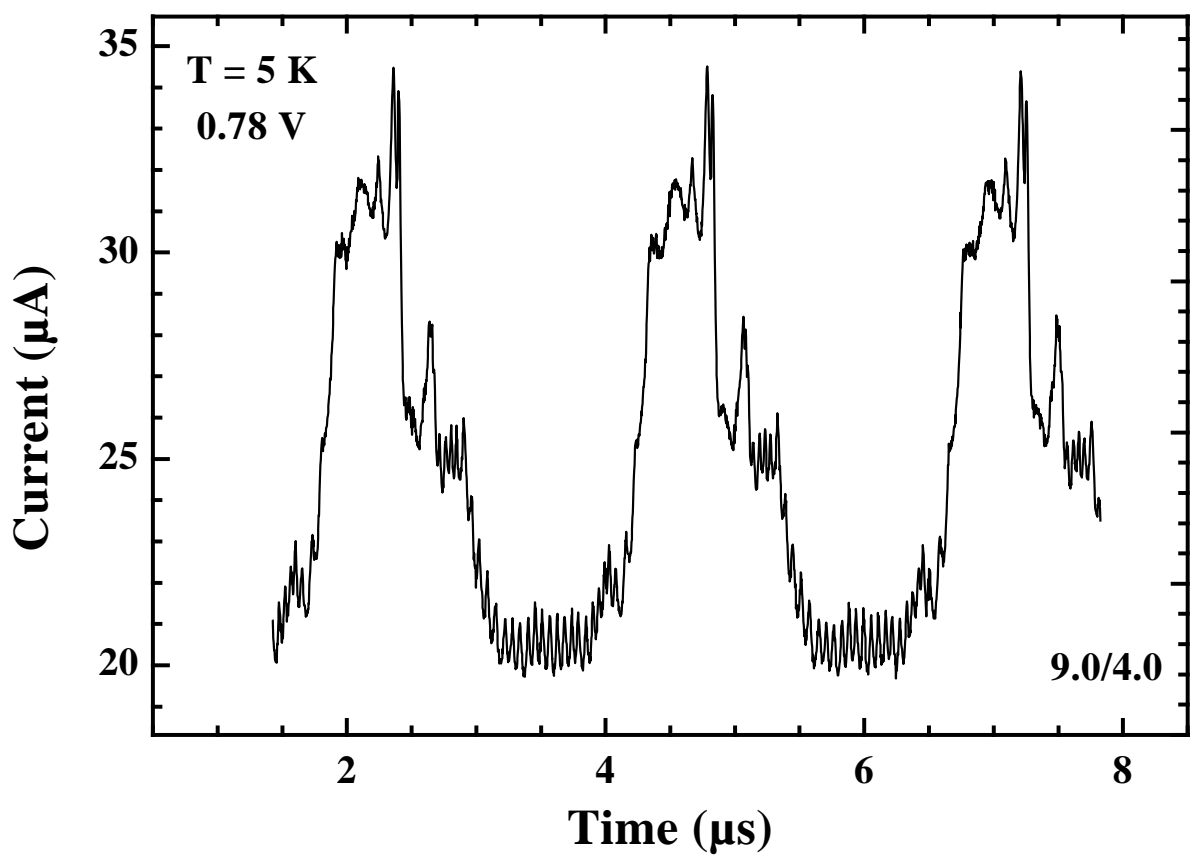

Fig. 4 


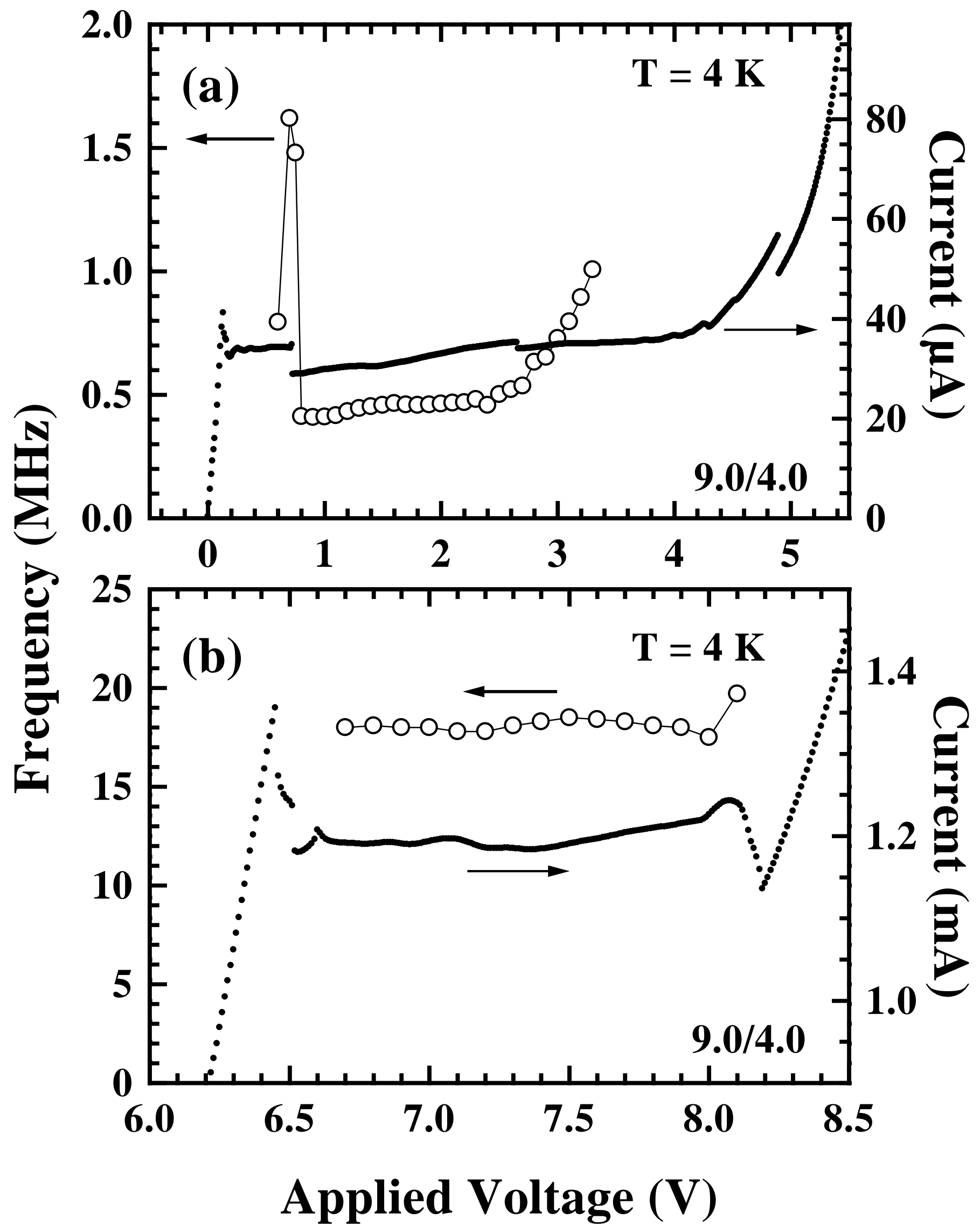

Fig. 5 


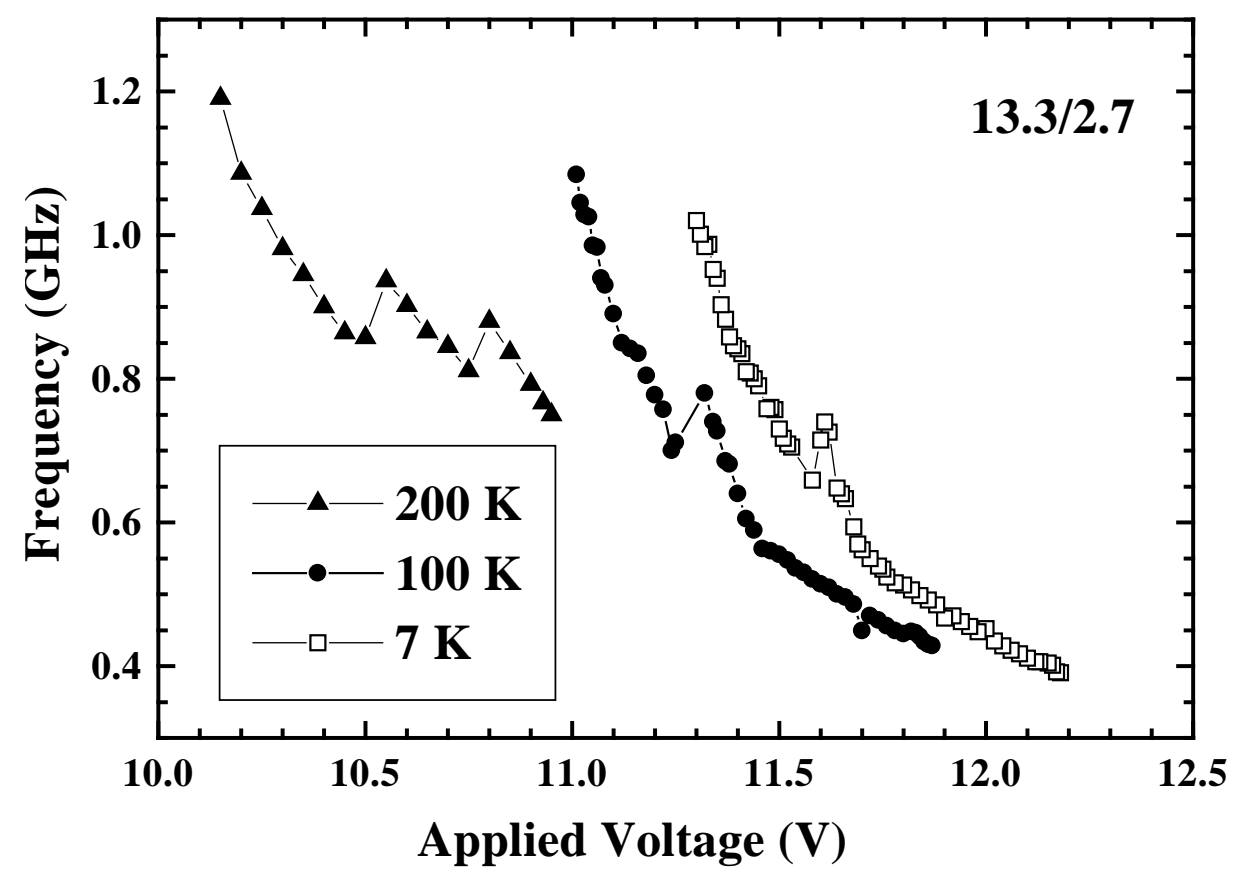

Fig. 6 


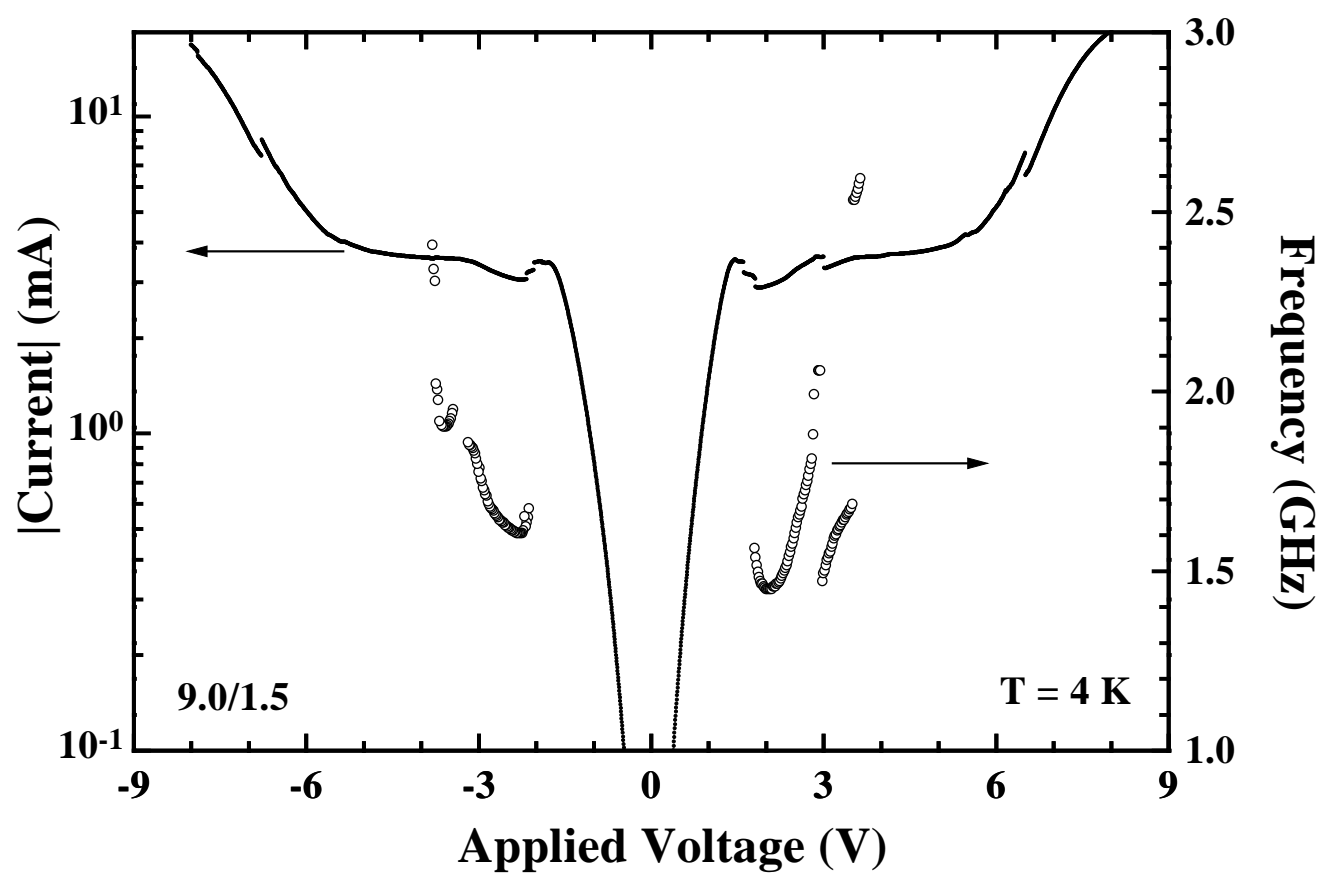

Fig. 7 


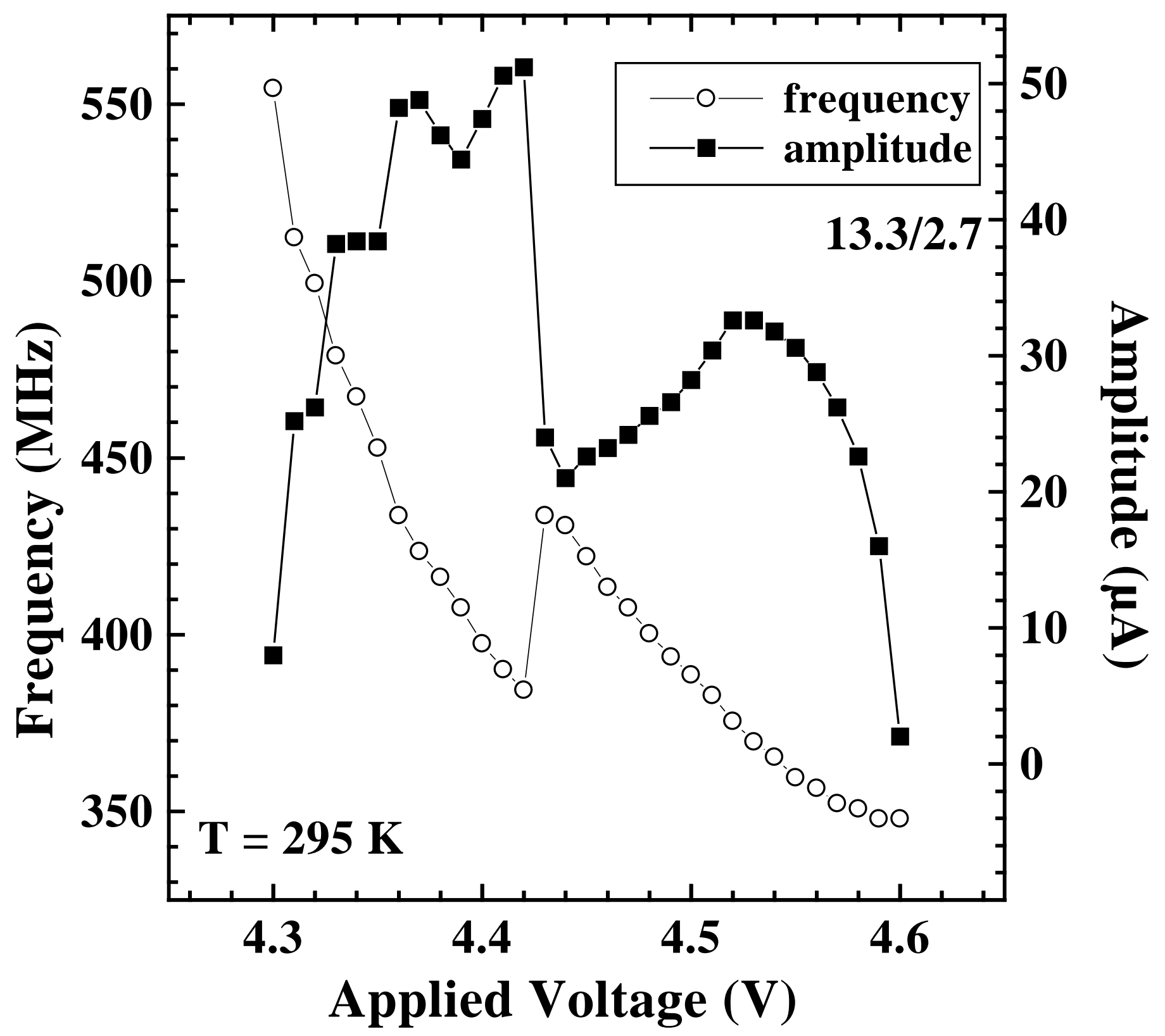

Fig.8 

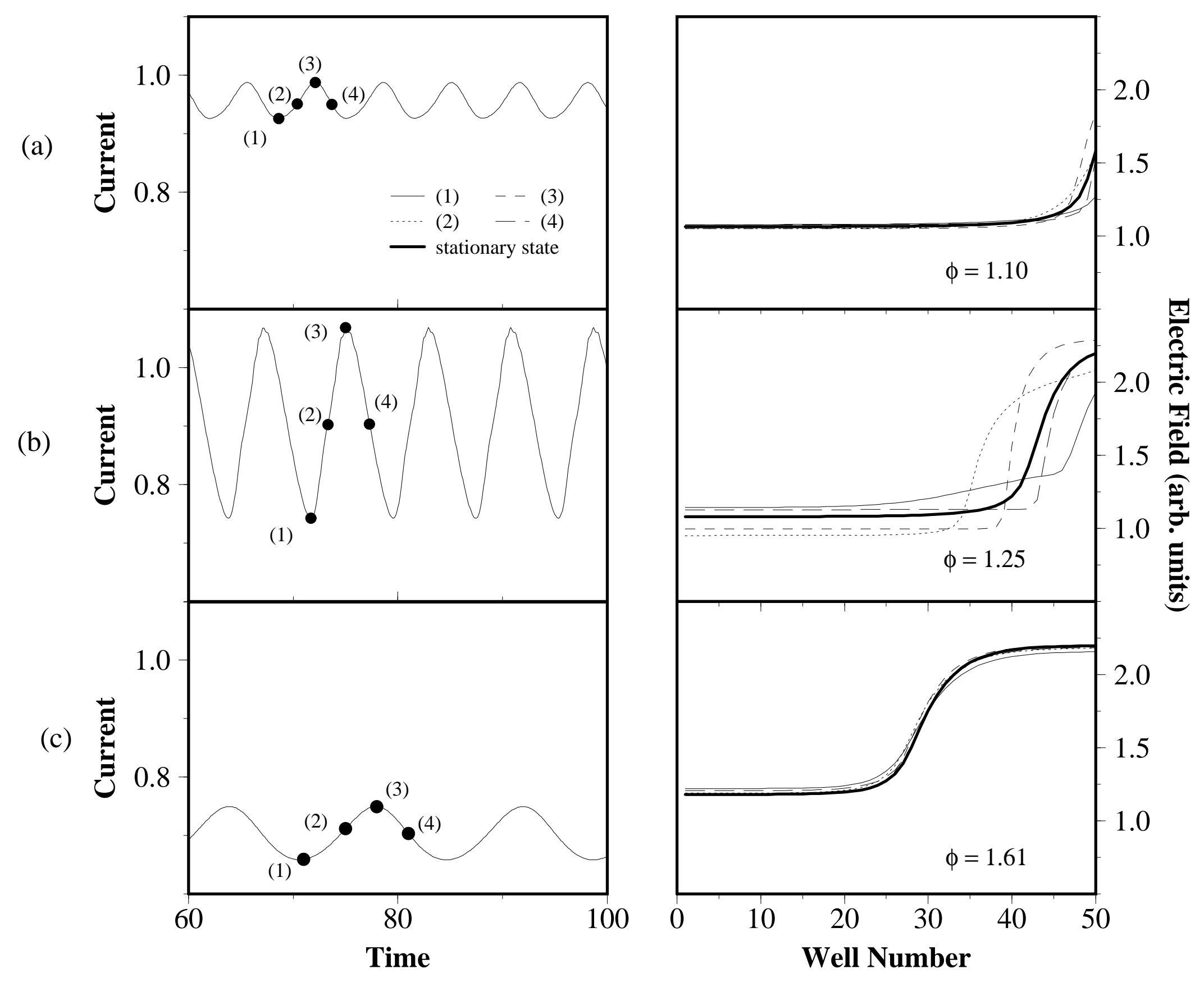


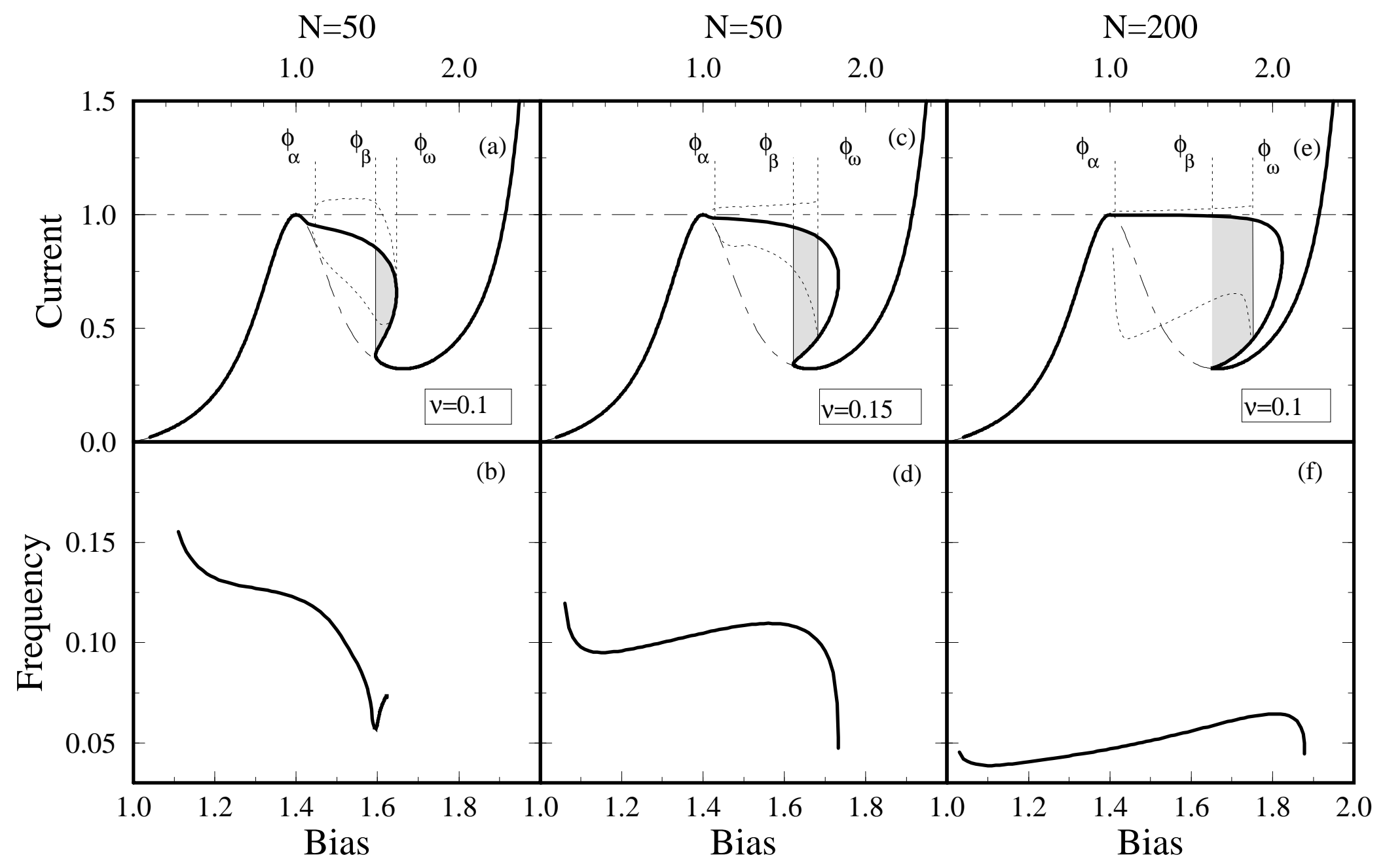




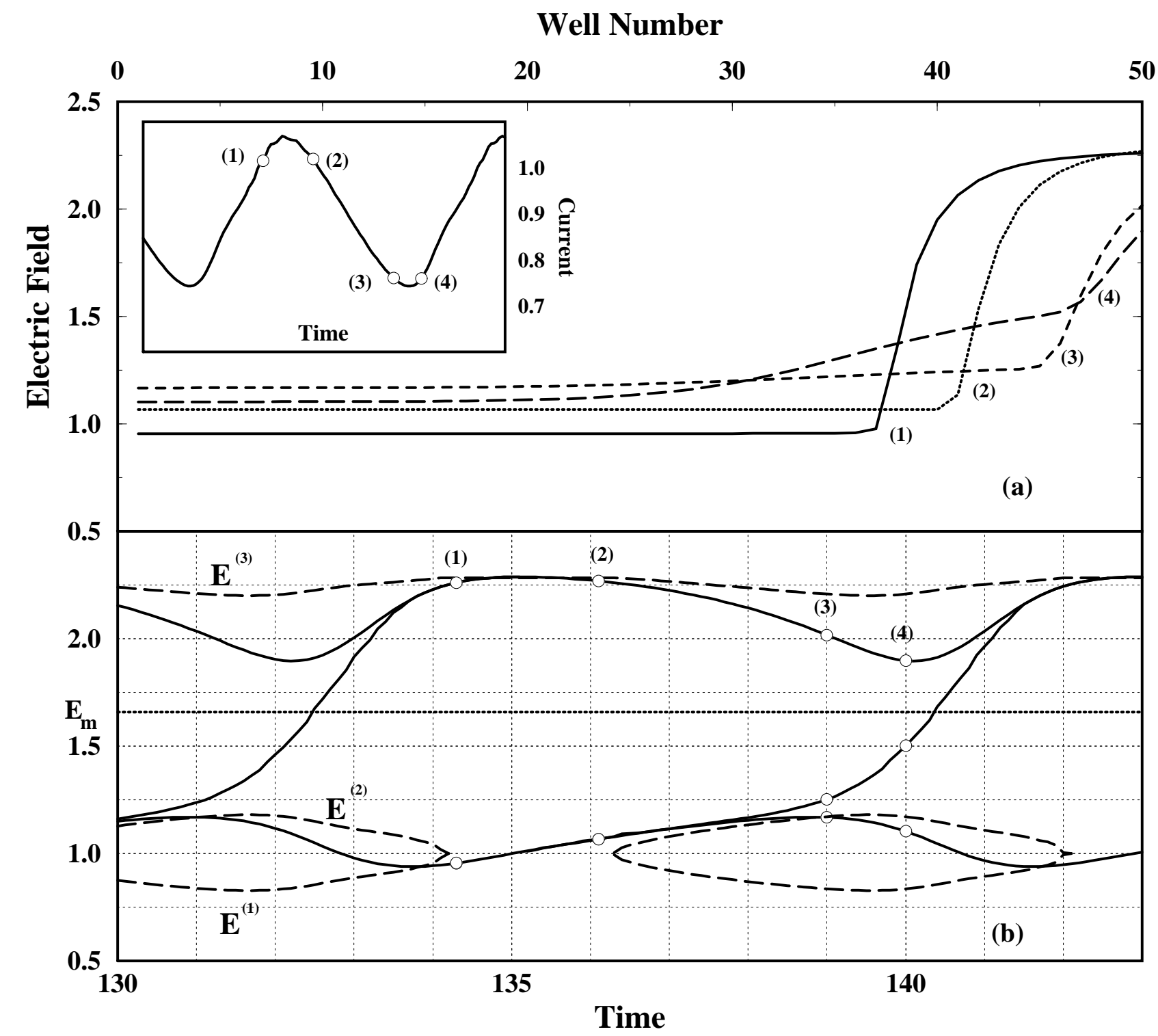




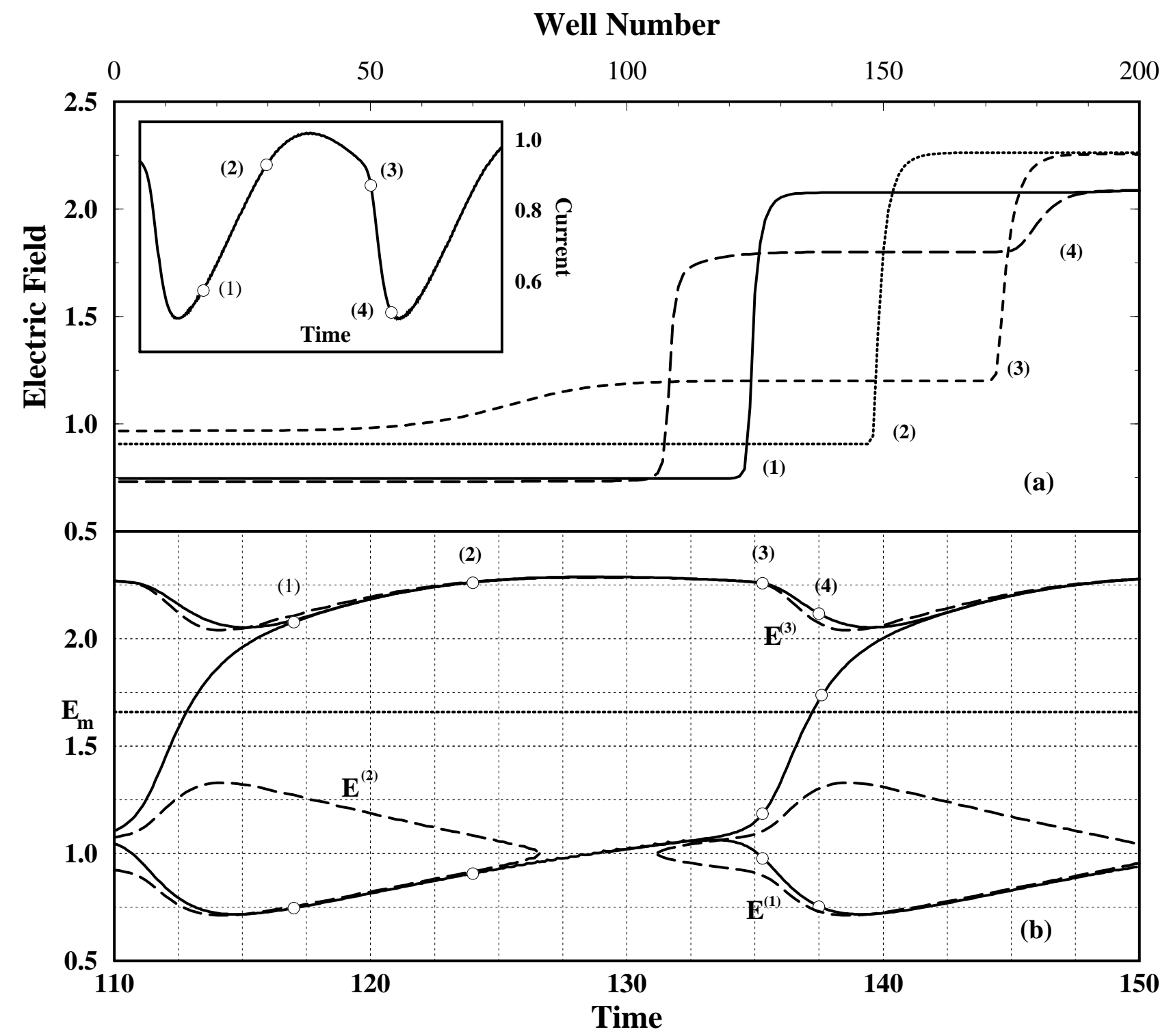



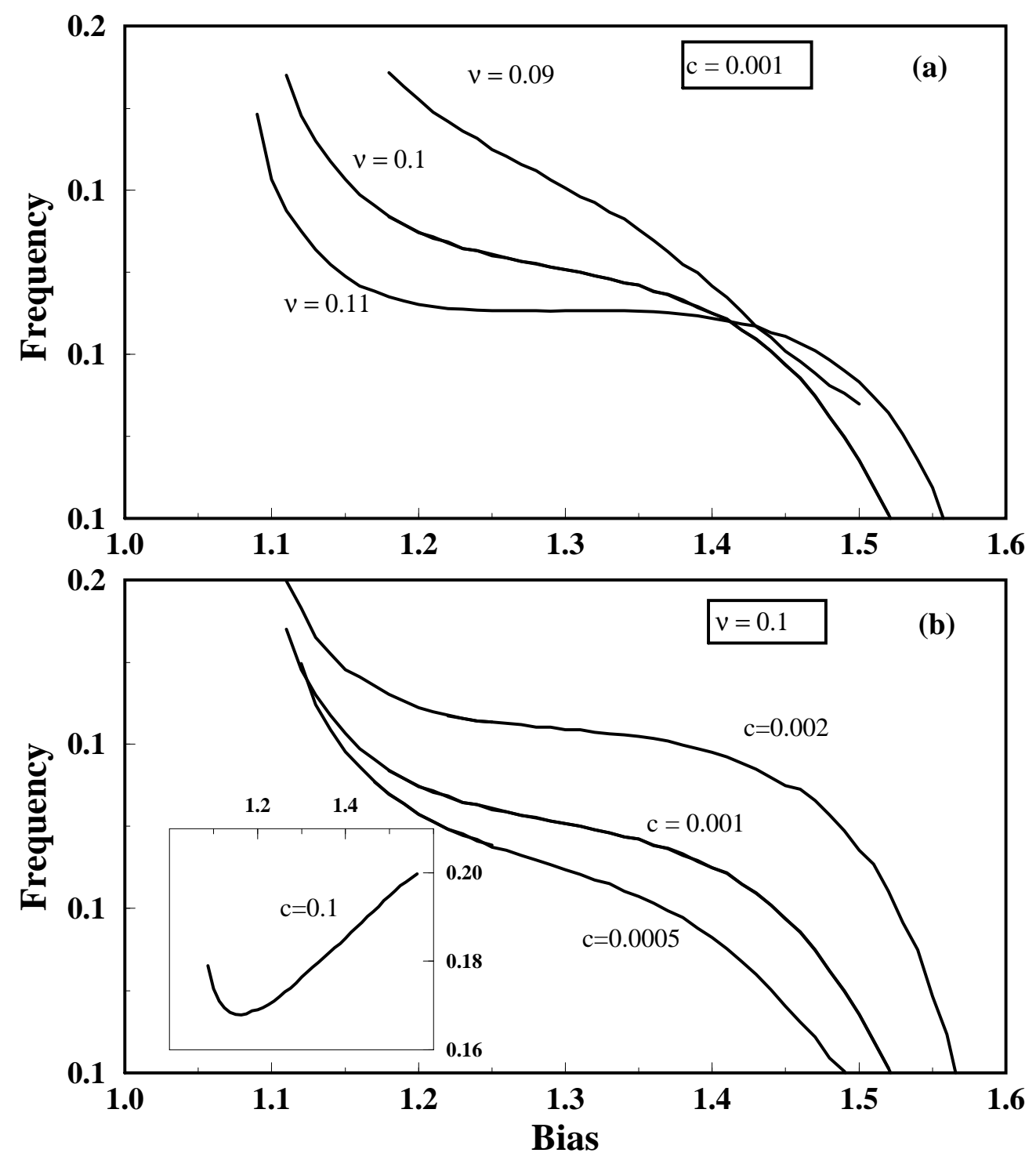

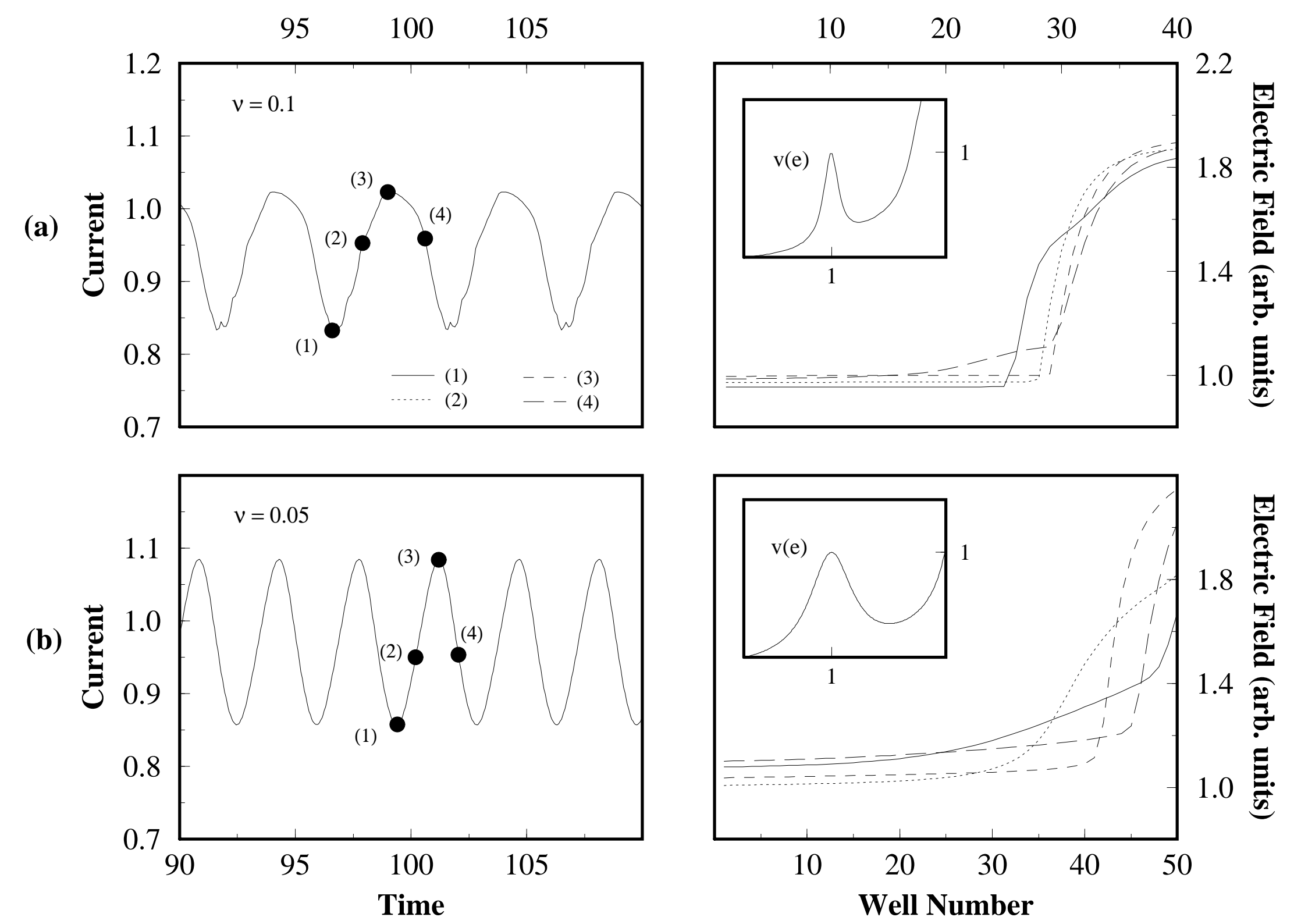
Time
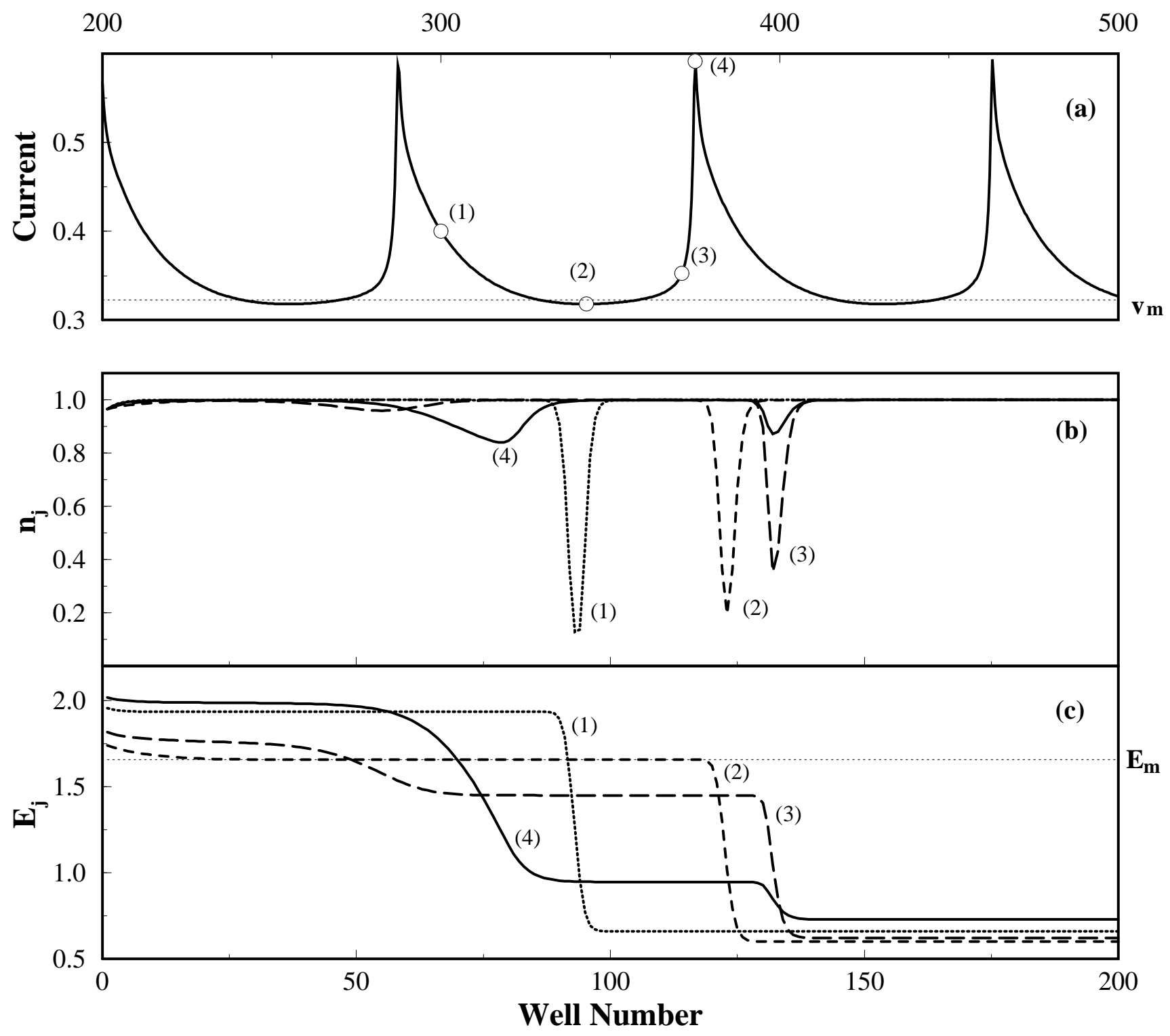


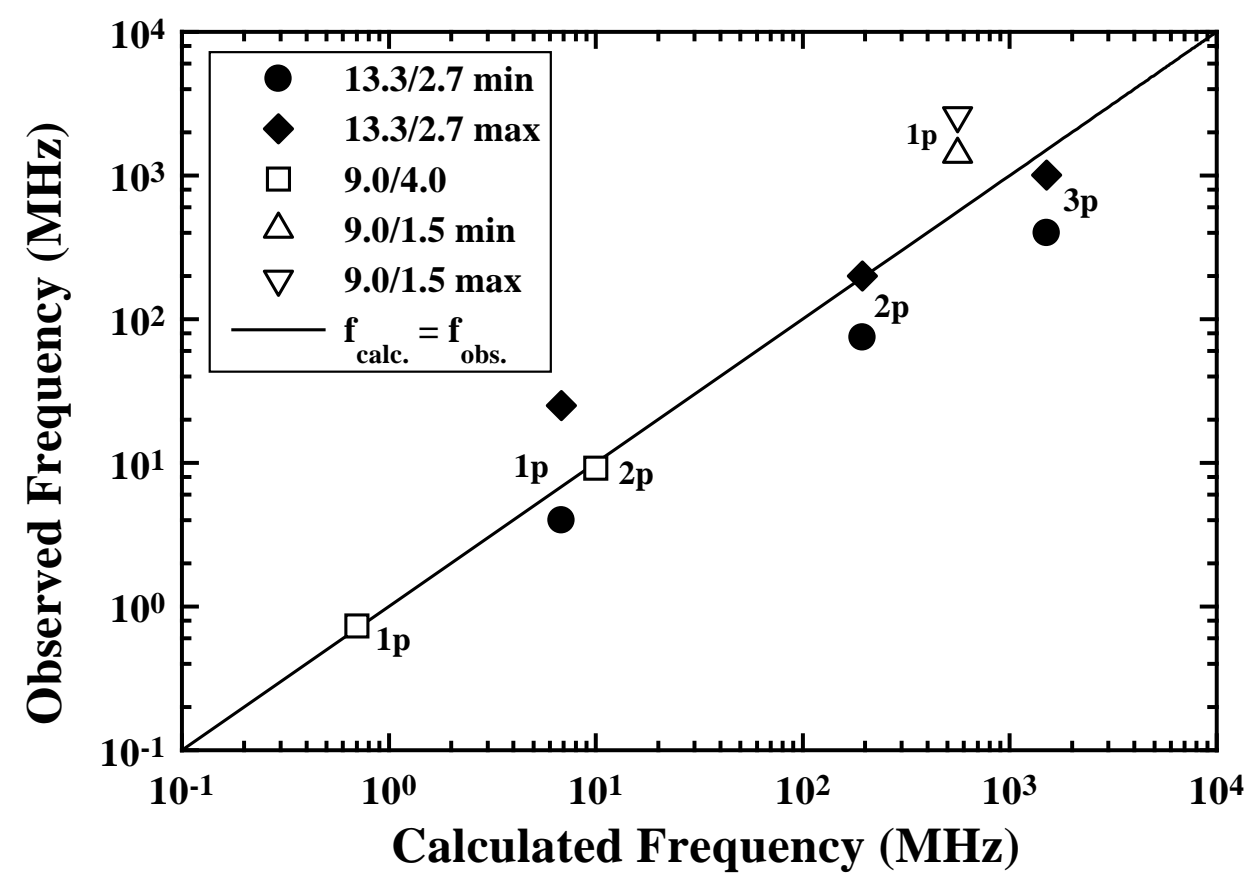

Fig.16 